\title{
NEEDLE VARIATIONS THAT CANNOT BE SUMMED*
}

\author{
ROSA-MARIA BIANCHINI ${ }^{\dagger}$ AND MATTHIAS KAWSKI ${ }^{\ddagger}$
}

\begin{abstract}
This article analyzes sets of higher order tangent vectors to reachable sets of analytic control systems (affine in the control). Both small-time local output controllability and small-time local controllability about a nonstationary reference trajectory are considered. In a series of purposefully constructed examples it is shown that the cones generated by needle variations may fail to be convex. The examples demonstrate that the usual technical condition that needle variations must be movable is essential to guarantee desirable convexity properties. Moreover, new doubts are cast on the structural stability of controllability properties, as apparently higher order perturbations can reverse the (lack of) controllability of lower order nilpotent approximating systems, thereby providing new insights about the ultimate question of whether controllability is finitely determined.
\end{abstract}

Key words. nonlinear controllability, optimality, control variations, tangent cones

AMS subject classifications. 93B05, 49K15

PII. S0363012902402876

1. Introduction. A fundamental dichotomy for a control system with a reference trajectory is whether the trajectory is optimal or whether the system is controllable about this trajectory. Topologically, this translates into the reference trajectory lying either on the boundary or in the interior of the funnel of reachable sets. Derivatives of the endpoint map with respect to the controls serve as the main analytic tool: If the derivative has full rank, then the trajectory lies in the interior and the system is controllable. Conversely, a necessary condition for optimality is that the derivative does not have full rank, which leads to the Pontryagin maximum principle [19] (which is a first derivative test). See [23] for the current state of the art unifying nonsmooth with differential geometric approaches.

Since commonly the first derivative does not have full rank (at the reference control), there have been many efforts to obtain higher order conditions for controllability and optimality, with Krener's high order maximal principle [17], Stefani's high order conditions for optimality [20], and Sussmann's general theorem for controllability [22] being a few prominent classical ones. See [23] and the references therein for the current state of the art. As opposed to purely theoretical statements in terms of high order Frechet derivatives, the main interest is in effectively computable conditions, leading, e.g., to algebraic rank conditions in terms of iterated Lie brackets of the system vector fields evaluated at a point as, e.g., in [20, 22]. Underlying such conditions are notions of higher order tangent vectors together with open mapping theorems. The higher order tangent vectors are basically high order directional derivatives of the endpoint

${ }^{*}$ Received by the editors February 21, 2002; accepted for publication (in revised form) October 10, 2002; published electronically March 26, 2003. A preliminary announcement of selected results of this paper has been presented as Lack of convexity for tangent cones of needle variations, 41st IEEE Conference on Decision and Control, Las Vegas, NV, 2002.

http://www.siam.org/journals/sicon/42-1/40287.html

†Dipartimento di Matematica, "Ulisse Dini," Università degli Studi di Firenze, Viale Morgagni 67a, 50134 Firenze, Italy (bianchin@math.unifi.it).

${ }^{\ddagger}$ Department of Mathematics and Statistics, Arizona State University, Tempe, AZ 85287-1804 (kawski@asu.edu). This author gratefully acknowledges the hospitality of the Universita degli Studi in Firenze and the support of the Italian CNR for this work. He also acknowledges the support of the NSF through grant DMS 00-72369. 
map, obtained from families of control variations (i.e., curves in the space of admissible controls). Open mapping theorems guarantee that if a cone of tangent vectors is the whole tangent space, then the reference trajectory lies in the interior. Of course, depending on the precise technical notion of the tangent object, one needs dedicated open mapping theorems - see, e.g., Lee and Markus [18] for such classical statements. A notable effort in the 1980s by Frankowska employed nonsmooth analysis to obtain a general purpose open mapping theorem $[9,10]$, relying on high order variations of set-valued maps.

The basic trade-off is between narrowly defined tangent objects (with straightforward open mapping theorems) and large tangent objects, which may lack nice convexity and approximation properties. Recall that classical calculus of variations primarily employed families of variations that are parameterized by their amplitude. In optimal control theory several alternative approaches have been analyzed. These include both curves $s \mapsto u_{s}(\cdot), s \geq 0$ (with $u_{0}=u^{*}$ the reference control) such that $\left\|u_{s}-u^{*}\right\|_{\infty} \searrow 0$, curves such that $\left\|u_{s}-u^{*}\right\|_{1} \searrow 0$ as $s \searrow 0$ (compare with [8]), and the so-called needle variations: Loosely speaking, these are such that the variation $u_{s}$ agrees with the reference control $u^{*}$ everywhere except on a (finite number of) interval(s) whose (combined) length goes to zero as $s \searrow 0$. The main attraction of such needle variations is that they are conceptually easy to combine for the purpose of generating convex combinations of tangent vectors, resulting in tangent cones with nice convexity properties. Of course, such schemes hinge on the intervals on which the variations differ from the reference control, eventually (for sufficiently small $s>0$ ) becoming disjoint. Alternatively, one might require that every needle variation be movable, i.e., one must be able to move the variation by a small time-amount and still be able to generate the same tangent vector. Precise technical specification of such conditions can be quite involved.

This article is not overly concerned about specific ways of combining needle variations. The main results are independent of such technical intricacies because we show that certain directions can be generated by the most basic needle variations, while certain other points cannot be reached by any control variations at all. Of course, this implies that one cannot reach those points or generate those tangent vectors by any kind of composition of needle variations either, no matter how these are defined.

For analytic, affine control systems with a stationary reference trajectory, no such conditions are needed, and a wealth of necessary and sufficient conditions for controllability and optimality have been found in recent decades; see, e.g., [22]. On the other hand, distinctive features of the existing literature for nonstationary reference trajectories are much more careful and provide more delicate notions of variations, tangent objects, and open mapping theorems; see, e.g., [1, 4, 5, 6, 16, 23]. A major open question has been whether such technical conditions are truly essential, or whether they are merely artifacts of a still imperfect knowledge level (e.g., future work might show that they are automatically satisfied for reasonable classes of systems). This article provides a definitive answer: Loosely speaking, even for the most benign classes of nonlinear systems (polynomial cascades, affine in the control, convex compact control values), the tangent cones generated by needle variations can be nonconvex. Moreover, even if their convex hull is the entire space the reference trajectory may lie on the boundary of the reachable sets. Both may happen even for arbitrary small positive times.

This article is organized as follows: After this introduction, we give and review technical definitions of controllability, needle variations, tangent cones, and open map- 
ping theorems. The subsequent three sections analyze a sequence of increasingly more delicate examples. First we consider the output controllability of a simple polynomial cascade system about an equilibrium (stationary reference trajectory). Most of the innovative constructions and critical inequalities may be found here. The next section slightly modifies the system so that it falls into the class of standard small-time local controllability about a nonstationary reference trajectory. Finally, we add some terms which at first sight appear to be higher order perturbations, but we show that these actually destroy the delicate controllability properties. The final section reflects and speculates on the possible implications of such a lack of robustness of the notion of controllability.

The constructions in the proofs fall into two categories: On one side we provide very explicit constructions of needle variations and demonstrate that they generate certain tangent vectors. Similarly, in the absence of applicable open mapping theorems, we explicitly construct controls that steer the system to any given target point. On the other side, we show that certain (regions of) points cannot be reached by any control whatsoever, and thus not by any needle variations either. Such arguments naturally involve delicate arguments with integral inequalities.

2. Control variations and approximating cones. The main thrust of this article is counterexamples. Thus for positive controllability results we use constructions that rely on small sets of tools, e.g., very narrow notions of (needle) variations. Such results clearly hold a fortiori if the sets of admissible variations etc. are broadened. Conversely, negative results (lack of convexity or controllability) are usually stated in the form that even for very large sets of controls something is impossible.

The systems under consideration are finite-dimensional, deterministic, analytic systems (possibly including an analytic output function) that are affine in the control.

$$
\dot{x}=f_{0}(x)+\sum_{i=1}^{\ell} u_{i}(t) f_{i}(x), \quad x(0)=0, \quad y=\varphi(x) .
$$

Here $x \in \mathbb{R}^{n}, f_{i}: \mathbb{R}^{n} \mapsto \mathrm{T} \mathbb{R}^{n}$ are analytic vector fields; the controls $u:[0, T] \mapsto U \subseteq \mathbb{R}^{\ell}$ are measurable functions defined on finite intervals and take values in a compact convex set $U$, usually $U=[-1,1]^{\ell}$. The output map $\varphi: \mathbb{R}^{n} \mapsto \mathbb{R}^{p}$ is analytic. In this article the vector fields are actually always of a polynomial cascade form, the controls in all constructions are piecewise constant, and the output map is either the identity or a projection onto a subspace. The solution curves of (2.1) corresponding to a control $u$, starting from $x(0)=0$, are denoted by $x(t, u)$ or, when no confusion arises, by $x(t)$. Their images under the output map are denoted $y(t, u)=\varphi(x(t, u))$ or simply $y(t)$. Throughout this article we also conveniently identify the tangent spaces $\mathrm{T}_{p} \mathbf{R}^{n}$ with $\mathbf{R}^{n}$.

The reachable sets $\mathcal{R}(t)$ consist of all points $x(t, u)$ reached in time $t$ by trajectories of (2.1) from $x(0)=0$ by means of admissible controls (measurable, and with values in $U$ almost everywhere). Given a reference trajectory $x^{*}(t)=x\left(t, u^{*}\right)$, controllability is defined as follows.

DEFINITION 2.1. The system (2.1) is small-time locally controllable (STLC) about $x^{*}$ if $x^{*}(t) \in \operatorname{int} \mathcal{R}(t)$ for all $t>0$. The system (2.1) is small-time locally output controllable (STLOC) about $y^{*}=\varphi\left(x^{*}\right)$ if $y^{*}(t) \in \operatorname{int}(\varphi(\mathcal{R}(t)))$ for all $t>0$.

The latter notion is related to Kaskosz's $g$-controllability [13] but is not yet standard. In general one needs to distinguish STLOC about a reference output $y^{*}$ with a fixed initial condition $x(0)=0$ from a notion that requires only $x(0) \in \varphi^{-1}\left(y_{0}\right)$. In 
this article this distinction will not play a role.

Throughout this article we will use the norm $\|x\|_{\infty} \stackrel{\text { def }}{=} \max _{k=1}^{n}\left|x_{k}\right|$ for $x \in \mathbb{R}^{n}$. In particular, the open ball of radius $r \geq 0$ about $p \in \mathbb{R}^{n}$ is $B_{p}^{\infty}(r)=\left\{x \in \mathbb{R}^{k}: \| x-\right.$ $\left.p \|_{\infty}<r\right\}$. Since $\|x\|_{\infty} \leq\|x\|_{2} \leq \sqrt{n}\|x\|_{\infty}$, this does not change the quality of any statements but allows us to dispense with lots of additional factors of $\sqrt{n}$ throughout our constructions and statements of results.

DeFinition 2.2. A one-parameter family of control variations of a reference control $u^{*}:[0, T] \mapsto U$ is a curve $s \mapsto u_{s} \in \mathcal{U}=\{u:[0, T] \mapsto U$ measurable $\}$ defined for $s \in\left[0, s_{0}\right]$ for some $s_{0}>0$ and with $u_{0}=u^{*}$. Such a family is called a family of control variations at zero if $u_{s}(t)=u^{*}(t)$ for all $t \in(s, T]$.

In the latter case it is convenient to identify $u_{s}$ with its restriction to $[0, s]$. Conversely we generally do not distinguish between a control $u_{s}:[0, s] \mapsto U$ and its extension to $[0, T]$ defined by setting $u_{s}(t)=u^{*}(t)$ if $t \in(s, T]$. Using either kind of curves one may define derivatives to the family of reachable sets. Denote by $\Phi:(t, p) \mapsto \Phi_{t}(p)=x\left(t, u^{*} ; p\right)$ the flow corresponding to the reference control $u^{*}$. In all systems analyzed in this article, this flow will be analytic, and hence there are no subtleties about transporting tangent vectors via the tangent maps $\Phi_{t *}$ from one tangent space to another. To facilitate comparisons, we move all tangent vectors back to the tangent space at the initial point. In the following definitions let $u^{*}:[0, T] \mapsto U$ be an admissible control generating a (reference) trajectory $x^{*}:[0, T] \mapsto \mathbf{R}^{n}$.

Definition 2.3. A vector $v \in \mathbb{R}^{n}$ is an mth order tangent vector to the family of reachable sets $\{\mathcal{R}(T)\}_{T \geq 0}$ at (time) zero, written $v \in \mathcal{K}_{0}^{m}$, if there exists a family of control variations at zero $u_{s}:[0, s] \mapsto U$ of $u^{*}$ such that

$$
x\left(s, u_{s}\right)=x\left(s, u^{*}\right)+s^{m} \Phi_{s *}(v)+o\left(s^{m}\right) .
$$

Definition 2.4. A vector $v \in \mathbb{R}^{p}$ is called an mth order tangent vector to the output-reachable sets of the system (2.1) with stationary reference trajectory $x^{*} \equiv 0$, written $v \in \mathcal{K}_{\varphi}^{m}$, if there exists a family of control variations $u_{s}:[0, s] \mapsto U$ such that

$$
\varphi\left(x\left(s, u_{s}\right)\right)=\varphi(x(0))+s^{m} v+o\left(s^{m}\right) .
$$

Most of the statements in this article involve these approximating sets to the family of reachable sets at the initial time. However, in a few places (e.g., system (2.9) and Corollary 5.8) it is convenient for precise statements to use the following notion of tangent vectors to a fixed reachable set (but transported back to the initial time for easy comparisons). In order to allow rudimentary comparisons with the above notions of higher order tangent vectors, the following definition uses the $L^{1}$-norm of the differences $\left(u_{s}-u^{*}\right)$.

DEFINITION 2.5. A vector $v \in \mathbb{R}^{n}$ is an mth order tangent vector to the reachable set $\mathcal{R}(T)$ at $x^{*}(T)$, written $v \in \mathcal{K}_{T}^{m}$, if there exists a family of control variations $u_{s}:[0, T] \mapsto U$ of $u^{*}$ such that

$$
x\left(T, u_{s}\right)=x\left(T, u^{*}\right)+\left\|u_{s}-u^{*}\right\|_{1}^{m} \Phi_{T *}(v)+o\left(\left\|u_{s}-u^{*}\right\|_{1}^{m}\right) .
$$

Note, that in all three definitions no regularity whatsoever is assumed or required for the maps $s \mapsto u_{s}$.

If $\left\{u_{s}\right\}_{s>0}$ is a family of control variations at zero (and thus $u_{s}(t)=u^{*}(t)$ for $s<t \leq T$ ), it is clear that $\left\|u_{s}-u^{*}\right\|_{1}^{m} \leq c^{m} s^{m}$, where $c=\max _{w \in U}|w|$ is finite (due to compactness of $U$ ). Thus, basically from the definition,

$$
\mathcal{K}_{0}^{m} \subseteq \mathcal{K}_{T}^{m} \quad \text { for all } m \geq 0 \text { and all } T>0 .
$$


However, the reverse inequality need not hold as, e.g., $\left\|u_{s}-u^{*}\right\|_{1}^{m} \searrow 0$ might go to zero faster than $s^{m}$ as $s \searrow 0$. Nonetheless, for typical families of control variations at zero, as constructed in what follows, one often can readily conclude that they generate corresponding tangent vectors of the same order $m$, lying in $\mathcal{K}_{0}^{m}$ and $\mathcal{K}_{T}^{m}$. Write $\overline{\mathcal{K}}_{T}$, $\overline{\mathcal{K}}_{0}^{m}$, and $\overline{\mathcal{K}}_{\varphi}^{m}$ for the cones $\overline{\mathcal{K}}_{0}^{m}=\left\{\lambda v: v \in \mathcal{K}_{0}^{m}, \lambda \geq 0\right\}$ etc. generated by $\mathcal{K}_{T}, \mathcal{K}_{0}^{m}$, and $\mathcal{K}_{\varphi}^{m}$, respectively, and also refer to the elements of the cones $\overline{\mathcal{K}}^{m}$ as tangent vectors. In the case of a stationary reference trajectory $x^{*} \equiv x(0)$ together with full-state output $\varphi \equiv \operatorname{id}_{\mathbf{R}^{\mathrm{n}}}$, the definitions of $\mathcal{K}_{0}^{m}$ and $\mathcal{K}_{\varphi}^{m}$ agree, and they are subsets of adjacent tangent cones and, moreover, have nice convexity and approximation properties [9].

The sets $\overline{\mathcal{K}}_{\varphi}^{m}$ of tangent vectors to output-reachable sets in general may have more complicated structures. In particular, they generally are not simply the images of the corresponding sets $\overline{\mathcal{K}}_{0}^{m}$ under the tangent map $\varphi_{*}$ as illustrated in the basic example

$$
\begin{cases}\dot{x}_{1}=u, & x(0)=0, \\ \dot{x}_{2}=x_{1}^{2}, & |u(\cdot)| \leq 1, \\ \dot{x}_{3}=x_{1}^{3}, & \varphi(x)=\left(x_{1}, x_{3}\right)\end{cases}
$$

with reference control $u^{*} \equiv 0$. Here $\overline{\mathcal{K}}_{0}^{m}=\mathbf{R} \times\{(0,0)\}$ for $m=1,2$, and $\overline{\mathcal{K}}_{0}^{m}=$ $\mathbf{R} \times[0, \infty) \times\{0\}$ for $m \geq 3$, while $\overline{\mathcal{K}}_{\varphi}^{m}=\mathbf{R}^{2}$ for $m \geq 4$. In particular, $\overline{\mathcal{K}}_{\varphi}^{4} \neq \varphi_{*} \overline{\mathcal{K}}_{0}^{4}$.

A key step to rendering the sets of tangent vectors useful for obtaining optimality results is to establish their convexity. For a wide variety of different settings, results that are similar to the following may be found in, e.g., $[4,5,6,8,9,10,14,18]$.

Proposition 2.1. For systems of form (2.1) with a stationary reference trajectory the following hold:

If $\lambda \in[0,1]$ and $v \in \mathcal{K}_{0}^{m}$, then $\lambda v \in \mathcal{K}_{0}^{m}$.

If $m \leq m^{\prime}$, then $\mathcal{K}_{0}^{m} \subseteq \mathcal{K}_{0}^{m^{\prime}}$.

If $v_{1}, v_{2} \in \mathcal{K}_{0}^{m}$ and $\lambda \in[0,1]$, then $\lambda^{m} v_{1}+(1-\lambda)^{m} v_{2} \in \mathcal{K}_{0}^{m}$.

TheOREm 2.2 (see $[9,10,11,14])$. Suppose $x^{*} \equiv 0$ is the stationary reference trajectory of a system of form (2.1). Then for every closed convex cone $K$ that is strictly contained in $\{0\} \cup \operatorname{int} \overline{\mathcal{K}}_{0}^{m}$, there exist $C>0$ and $T>0$ such that

$$
K \cap B_{0}^{\infty}\left(C t^{m}\right) \subseteq \mathcal{R}(t) \text { for all } 0 \leq t \leq T .
$$

Moreover, if $\overline{\mathcal{K}}_{0}^{m}=\mathbb{R}^{n}$, then the system (2.1) is $S T L C$ about $x^{*}=0$, and the minimum time function $V(q)=\min \{t \geq 0: \exists u$ such that $x(t, u)=q\}$ is Hölder continuous of order $\frac{1}{m}$ at $x=0$.

If the reference trajectory is nonstationary, then the cones $\overline{\mathcal{K}}_{0}^{m}$ need not have similarly nice convexity and approximation properties. Thus much work in recent decades has focused on developing more refined notions of tangent objects for that setting. The classical open mapping theorems (compare, e.g., $[18,19])$ use topological arguments that rely on continuity of the multiparameter families of control variations that naturally generalize the (single-parameter) curves introduced above. Typically, one might have constructed $n$ curves $s \mapsto u_{s}^{(i)} \in \mathcal{U}$, which generate the tangent vectors $v^{(i)} \in \mathcal{K}_{0}^{m}, i=1, \ldots, n$. The critical next step is to somehow combine these variations in order to show that the convex combinations $\left(c_{1} v_{1}+\cdots+c_{n} v_{n}\right)$ with $c_{1}+\cdots+c_{n}=1$ are $m$ th order tangent vectors, i.e., are contained in $\overline{\mathcal{K}}_{0}^{m}$, too. A natural first try is to consider convex combinations of suitable reparameterizations of the original curves

$$
s \mapsto u_{s, c_{1}, \ldots, c_{n}}=c_{1} u_{\alpha_{1}(c, s)}^{(1)}+c_{2} u_{\alpha_{2}(c, s)}^{(2)}+\cdots+c_{n} u_{\alpha_{n}(c, s)}^{(n)}
$$


and analyze the corresponding curves $s \mapsto x\left(s, u_{s, c}\right)$ of endpoints for each value $c$. Variations that lend themselves especially well to generating such convex combinations are needle variations. (Alternatively, compare with, e.g., [8] for variations such that $\left\|u_{s}-u^{*}\right\|_{1} \searrow 0$.) Basically, each such control $u_{s}$ agrees with $u^{*}$ on all of $[0, T]$ except on an interval whose length is of order $s$ for $s \searrow 0$. Two such families of variations are easily combined unless the intervals on which they disagree from $u^{*}$ overlap for all small $s>0$. In that case, one might be tempted to simply move the interval in one of the families. The continuous dependence of solution curves on the data suggests that after such small (or vanishing, as $s \searrow 0$ ) moves, the combined variations might still generate the desired tangent vectors.

Before giving a technical (broad) definition of needle variations, we discuss a very simple example that illustrates the problem that some (narrowly defined) needle variations might not be able to be moved:

$$
\left\{\begin{aligned}
\dot{x}_{0} & =1, & |u(\cdot)| & \leq 1, \\
\dot{x}_{1} & =u, & x(0) & =0, \\
\dot{x}_{2} & =\left(x_{0}-1\right) x_{1}^{2}, & x^{*}(t) & =(t, 0,0,0), \\
\dot{x}_{3} & =x_{1}^{7}, & t & \in[0,2]=[0, T] .
\end{aligned}\right.
$$

In this case the family of needle variations $u_{s}^{ \pm}:[0,2] \mapsto[-1,1]$ defined by $u_{s}^{ \pm}(t)= \pm 1$ if $1-\frac{1}{2} s \leq t<1, u_{s}^{ \pm}(t)=\mp 1$ if $1 \leq t<1+\frac{1}{2} s$, and $u_{s}^{ \pm}(t)=0$ else, generates the curves $x\left(2, u_{s}^{ \pm}\right)=\left(2,0,0, \pm 2^{-10} s^{8}\right) \in \mathcal{R}_{0}(2)$, and thus $v=(0,0,0, \pm 1) \in \overline{\mathcal{K}}_{2}^{8}$ as 8 th order tangent vectors at $T=2$ (note that $\left\|u_{s}-u^{*}\right\|_{1}=s$ ).

In general, suppose $u_{s}:[0,2] \mapsto[-1,1]$ is any family of control variations that agrees with $u_{s}(t)=u^{*}(t)=0$ for all $t \in[0, T] \backslash I_{s}$, where $I_{s} \subseteq[0, T]$ is an interval of any of the forms $[a, a+s],[a-s, a]$, or $\left[a-\frac{s}{2}, a+\frac{s}{2}\right]$ for some fixed $a \in[0,2]$. Then $x\left(T, u_{s}\right)=\left(2,0,0, C s^{8}\right)+o\left(s^{8}\right)$ is only possible if $I_{s}$ is of the third form, and in addition $a=1$. This means that the only single family of needle variations that can generate $v=(0,0,0, \pm 1)$ as tangent vector "cannot be moved."

In this simple example it is clear that either $v$ can easily be generated by control variations whose support (technically the support of $\left(u_{s}-u^{*}\right)$ ) consists of two disjoint intervals, e.g.,

$$
u_{s}(t)=\left\{\begin{array}{cl}
1 & \text { if } \pm \frac{1}{2}-\frac{1}{4} s \leq t< \pm \frac{1}{2} \\
-1 & \text { if } \pm \frac{1}{2} \leq t< \pm \frac{1}{2}+\frac{1}{4} s \\
0 & \text { else. }
\end{array}\right.
$$

This demonstrates that if one considers only control variations that are supported in a single interval shrinking to a point, then it may be possible that certain tangent vectors can be generated only at a specific point $t_{0}$ that cannot be moved.

The example appears contrived and the notion of needle variation unnecessarily constrained. It is customary to broaden the notion of needle variations to allow the support of $\left(u_{s}-u^{*}\right)$ to be a finite union of intervals of total length of order $s$.

DeFINITION 2.6. A family of control variations $s \mapsto u_{s}:[0, T] \mapsto U$ defined for $s \in\left[0, s_{0}\right]$ with $s_{0}>0$ is a (family of) needle variations of the reference control $u^{*}=u_{0}$ if there exist a constant $C>0$ and a finite number of pairs of increasing functions $s \mapsto a_{s}^{(k)}$ and decreasing functions $s \mapsto b_{s}^{(k)}$ defining intervals $\left[a_{s}^{(k)}, b_{s}^{(k)}\right] \subseteq[0, T]$, $k=1, \ldots, N$, such that

$$
\operatorname{supp}\left(u_{s}-u^{*}\right) \subseteq \bigcup_{k=1}^{N}\left[a_{s}^{(k)}, b_{s}^{(k)}\right] \text { and } \sum_{k=1}^{N}\left(b_{s}^{(k)}-a_{s}^{(k)}\right) \leq C s \text { for all } s \leq s_{0} .
$$


Note that a family of variations at zero as defined earlier automatically qualifies as a family of needle variations as one may take $N=1$ and intervals defined by $a_{s}^{(1)}=0$ and $b_{s}^{(1)}=s$. Definition 2.6 also includes other common constructions with $N=1$ and $\left[a_{s}^{(1)}, b_{s}^{(1)}\right]=[\alpha, \alpha+s]$ (right variations at $\left.t=\alpha\right)$ or $\left[a_{s}^{(1)}, b_{s}^{(1)}\right]=[\beta-s, \beta]$ (left variations at $t=\beta$ ). The constructions in this article use mainly (right) needle variations at zero, combined with some (left) needle variations at the final time $T$. But the main claim of "needle variations that cannot be summed" holds even if the combinations (or sums) are allowed to lie in a more general class as in Definition 2.6.

3. The main construction. This section analyzes a custom-designed polynomial cascade system (affine in the control) with output, which forms the heart of the promised counterexample in section 5 . We demonstrate that the growth rates of its reachable sets are very sensitive to reflections about the origin. More specifically, the approximating cones of tangent vectors are described not only by intersections, but also by unions of half spaces. The basic system with output $\varphi$ is

$$
\left\{\begin{array}{lrl}
\dot{x}_{1}=u_{1}, & \left|u_{1}(\cdot)\right| \leq 1, \\
\dot{x}_{2}=u_{2}, & \left|u_{2}(\cdot)\right| \leq 1, \\
\dot{x}_{3}=x_{1}^{2}, & x(0)=0, \\
\dot{x}_{4}=x_{2}^{2}, & \varphi(x)=\left(x_{1}, x_{2}, x_{5}, x_{6}\right), \\
\dot{x}_{5}=x_{4} x_{1}^{2}-x_{1}^{7}, & \\
\dot{x}_{6}=x_{3} x_{2}^{2}-x_{2}^{7} . &
\end{array}\right.
$$

Figure 3.1 pictorially summarizes key properties of this system, showing crosssections of approximating cones of the image $\varphi(\mathcal{R}(t))$ of the reachable set under the output map. The remainder of this section proves technical statements that make this illustration precise.

Theorem 3.1. For all $T>0$ (sufficiently small)

$$
\varphi\left(R_{0}(T)\right) \supseteq B_{0}^{\infty}\left(2^{-18} T^{8}\right) \cap\left\{y \in \mathbb{R}^{4}:\left(y_{3} \geq 0 \text { or } y_{4} \geq 0\right) \text { and } y_{1}=y_{2}=0\right\} .
$$

The proof shows in particular that points of the forms $x=\left(0,0, *, *,-2^{-18} s^{8}, 0\right)$ and $x=\left(0,0, *, *, 0,-2^{-18} s^{8}\right)$ can be reached in time $s$, i.e., by control variations at zero. Thus the tangent vectors $(0,0,-1,0),(0,0,0,-1) \in \overline{\mathcal{K}}_{\varphi}^{8}$ are generated by needle variations of the zero reference control.
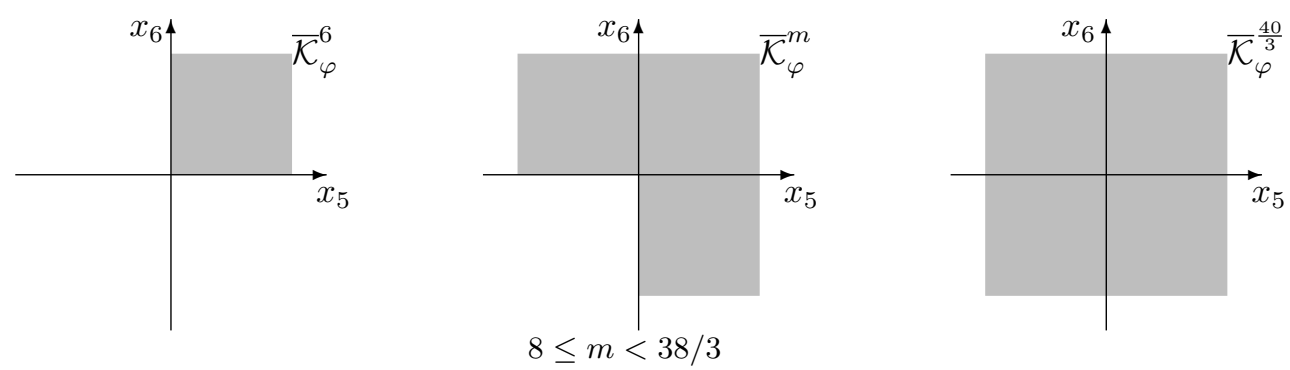

FIG. 3.1. Cross-sections of approximating cones of tangent vectors for system (3.1). 
Proof. Suppose $T>0$ and $\eta \in \mathbb{R}^{4}$ are given, with $\|\eta\|_{\infty}<2^{-18} T^{8}, \eta_{3}<0$, and $\eta_{4} \geq 0$. (The case of $\eta_{4}<0$ and $\eta_{3} \geq 0$ is analogous.) Set $t_{1}=\left(-4 \eta_{3}\right)^{1 / 8}>0$. Define the control $u:[0, T] \mapsto[-1,1]^{2}$ (with $t_{2} \geq 0$ to be determined later) by

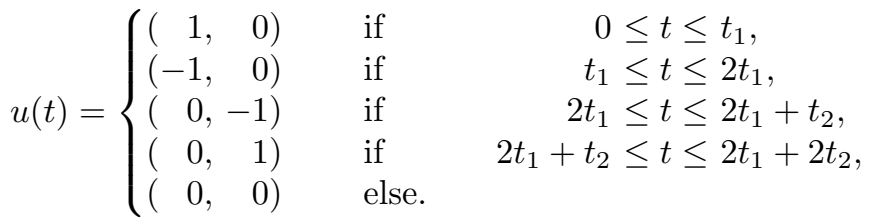

From $u_{2}(t)=0$ for $t \leq 2 t_{1}$ it is easily seen that $x\left(2 t_{1}, u\right)=\left(0,0, \frac{2}{3}\left(-4 \eta_{3}\right)^{3 / 8}, 0, \eta_{3}, 0\right)$ and thus $\varphi\left(x\left(2 t_{1}, u\right)\right)=\left(0,0, \eta_{3}, 0\right)$. Then $\varphi\left(x\left(2 t_{1}+2 t_{2}, u\right)\right)=\left(0,0, \eta_{3}, \xi\left(t_{2}\right)\right)$, where

$$
\xi: t_{2} \mapsto x_{6}\left(2 t_{1}+2 t_{2}\right)=2 \int_{0}^{t_{2}}\left(\frac{2}{3}\left(-4 \eta_{3}\right)^{3 / 8} t^{2}+t^{7}\right) d t=\frac{4}{9}\left(-4 \eta_{3}\right)^{3 / 8} t_{2}^{3}+\frac{1}{4} t_{2}^{8}
$$

is a strictly increasing function. Thus there exists a unique $0 \leq t_{2}^{*} \leq\left(4 \eta_{4}\right)^{1 / 8}$ such that $\xi\left(t_{2}^{*}\right)=\eta_{4}$. One easily verifies that the total time satisfies

$$
\left(2 t_{1}+2 t_{2}^{*}\right) \leq 2\left(-4 \eta_{3}\right)^{1 / 8}+2\left(4 \eta_{4}\right)^{1 / 8} \leq 4 \cdot\|4 \eta\|_{\infty}^{1 / 8}=\left(2^{18}\|4 \eta\|_{\infty}\right)^{1 / 8}<T
$$

Points $\eta=\left(0,0, \eta_{3}, \eta_{4}\right)$ with $\eta_{3}>0$ and $\eta_{4}>0$ are even easier to reach using again piecewise constant controls, but now both $u_{1}$ and $u_{2}$ start with value +1 , followed by -1 and zero. Note that in the directions of this positive quadrant the reachable set grown even faster, of order $T^{6}$.

Several stronger statements appear possible, e.g., characterizing the image $\varphi\left(\mathcal{R}_{0}(T)\right)$ of the reachable set without the condition $y_{1}=y_{2}=0$. However, in what follows we have no need for this, and it is quite cumbersome to find the precise complete boundary of the reachable sets.

One consequence of the next statement is that there does not exist a constant $C>0$ such that one can reach points $x=\left(0,0, *, *,-C T^{8},-C T^{8}\right)$ in the fourth quadrant for all sufficiently small times $T>0$ using any controls. Thus, a fortiori, one cannot reach such points by any combination of needle variations in time $T>0$, and $(0,0,-1,-1) \notin \overline{\mathcal{K}}_{\varphi}^{8}$.

Theorem 3.2. Suppose $0 \leq T<1$, and $x_{1}(T, u)=x_{2}(T, u)=0$. Define $C=3^{1 / 5} \cdot 2^{-35 / 3}>0$. If $x_{5}(T, u)<0$, then $x_{6}(T, u)>-C T^{38 / 3}$, and if $x_{6}(T, u)<0$, then $x_{5}(T, u)>-C T^{38 / 3}$.

The following useful restatements and consequences are immediate corollaries from the theorem (or from its proof given in what follows).

COROLlary 3.3. If $C, m>0$ are such that the images of reachable set of system (3.1) contain the open balls $B_{0}^{\infty}\left(C T^{m}\right) \subseteq \varphi\left(\mathcal{R}_{0}(T)\right.$ ) for all $T>0$ (sufficiently small), then $m \geq \frac{38}{3}$.

Corollary 3.4. For system (3.1), $(0,0, a, b) \in \overline{\mathcal{K}}_{\varphi}^{8}$ if and only if $a \geq 0$ or $b \geq 0$.

Proof of Corollary 3.4. The "if" part is clear from Theorem (3.1). For the "only if " part, suppose $(0,0, a, b) \in \mathcal{K}_{\varphi}^{8}$ for some $a, b \in \mathbb{R}$. By definition of $\mathcal{K}_{\varphi}$ there exist $0<s_{0}<1$ and a family of control variations $u_{s}:[0, s] \mapsto[-1,1]^{2}, 0 \leq s \leq s_{0}$, such that for some $\xi:\left[0, s_{0}\right] \mapsto \mathbb{R}^{6}$ with $\|\xi(s)\|_{\infty}=o\left(s^{8}\right)$ the endpoints have the form

$$
x\left(s, u_{s}\right)=s^{8} \cdot(0,0, *, *, a, b)+\xi(s) \in \mathcal{R}(s) .
$$


Define a new family of control variations $v_{2 s}:[0,2 s] \mapsto[-1,1]^{2}, 0 \leq 2 s \leq s_{0}$, by

$$
v_{2 s}(t)=\left\{\begin{aligned}
u_{s}(t) & \text { if } 0 \leq t \leq s \\
-\frac{1}{s} \cdot\left(\xi_{1}(s), \xi_{2}(s)\right) & \text { if } s<t \leq 2 s
\end{aligned}\right.
$$

Then $x\left(t, v_{2 s}\right)=x\left(t, u_{s}\right)$ for $0 \leq t \leq s$, and in particular $x\left(s, v_{2 s}\right)=x\left(s, u_{s}\right)$. For $t \in[s, 2 s]$ in the second half of the domain of $v_{2 s}$ the first two components $x_{i}\left(t, v_{2 s}\right)=$ $(2 s-t) \xi_{i}(s), i=1,2$, remain of size $o\left(s^{8}\right)$. Consequently also $x_{3}\left(t, v_{2 s}\right)$ and $x_{4}\left(t, v_{2 s}\right)$ remain of size $o\left(s^{8}\right)$, and the change in the last two components

$$
\left\|x_{i}\left(2 s, v_{2 s}\right)-x_{i}\left(s, v_{2 s}\right)\right\|_{\infty}=o\left(s^{24}\right) \text { for } i=5,6
$$

is again of higher order. The endpoints are of the form

$$
x\left(2 s, v_{2 s}\right)=\left(0,0, *, *, s^{8} a+o\left(s^{8}\right), s^{8} b+o\left(s^{8}\right)\right),
$$

and in particular they lie in the plane $x_{1}=x_{2}=0$, satisfying the hypotheses of Theorem 3.2. Thus $a \geq 0$ or $b \geq 0$.

Corollary 3.5. For $8<m<38 / 3$ the approximating cones $\overline{\mathcal{K}}_{\varphi}^{m}$ of the outputreachable set are not convex.

To streamline the proof of Theorem 3.2 we first establish two technical lemmata. Consider the following simple system about its equilibrium point $x=0$ :

$$
\left\{\begin{aligned}
\dot{x}_{1} & =u, & |u(\cdot)| & \leq 1, \\
\dot{x}_{2} & =c x_{1}^{2}-x_{1}^{7}, & x(0) & =0 .
\end{aligned}\right.
$$

Lemma 3.6. If $c \neq 0$, then the system (3.10) is not STLC about $x=0$. If $T>2\left(\frac{8}{3}|c|\right)^{\frac{1}{5}}$, then $0 \in \operatorname{int} \mathcal{R}(T)$.

Proof. W.l.o.g. assume $c>0$ (else consider the coordinate change $\left(x_{1}, x_{2}\right) \mapsto$ $\left.\left(x_{1},-x_{2}\right)\right)$. The first assertion follows from $x_{2}(T)=\int_{0}^{T} x_{1}^{2}(t)\left(c-x_{1}^{5}(t)\right) d t \geq 0$ since $\left|x_{1}^{5}(t)\right| \leq t^{5} \leq T^{5} \leq c$ if $T \leq c^{\frac{1}{5}}$.

Conversely, suppose $T>2 b_{0}$, where $b_{0}=\left(\frac{8}{3} c\right)^{\frac{1}{5}}$ is the first positive zero of $t \mapsto$ $\frac{1}{3} c t^{3}-\frac{1}{8} t^{8}$.

Consider the 2-parameter family of controls

$$
u_{a, b}(t)=\left\{\begin{aligned}
0 & \text { if } & 0 \leq t \leq T-2 b_{0}-2 b-a, \\
1 & \text { if } & T-2 b_{0}-2 b-a<t \leq T-b_{0}-b-a, \\
-1 & \text { if } & T-b_{0}-b-a<t \leq T .
\end{aligned}\right.
$$

Since $T-2 b_{0}>0$, these controls are defined for $(a, b)$ in some neighborhood of $(0,0)$. (To be precise, they are defined for $-b_{0} \leq b,-b_{0} \leq a+b$, and $a+2 b \leq T-2 b_{0}$.) It is straightforward to verify that $x\left(T, u_{0,0}\right)=0$ and that the Jacobian (for some polynomial $p$ in two variables)

$$
\frac{\partial x\left(T, u_{a, b}\right)}{\partial(a, b)}=\left(\begin{array}{cc}
-1 & 0 \\
c a^{2}+a^{7} & -\frac{20}{9}\left(54 c^{7}\right)^{\frac{1}{5}}+b \cdot p\left(b, c^{\frac{1}{5}}\right)
\end{array}\right)
$$

of the map $(a, b) \mapsto x\left(T, u_{a, b}\right)$ has full rank at $(a, b)=(0,0)$ when $(c \neq 0)$. By the inverse mapping theorem, $0 \in \operatorname{int} \mathcal{R}(T)$, and the system (3.6) is STLC.

Lemмa 3.7. Suppose $f:[0, T] \mapsto \mathbb{R}$ is absolutely continuous with $\left|f^{\prime}(t)\right| \leq \varepsilon$ a.e. and $f(0)=f(T)=0$. Then for any even integer $m=2 k \geq 2$

$$
\int_{0}^{T} f^{m}(t) d t \geq \frac{2}{(m+1) \varepsilon}\|f\|_{\infty}^{m+1}
$$


Proof. Since $f$ is continuous there exists $t_{0} \in[0, T]$ such that $f\left(t_{0}\right)=\|f(t)\|_{\infty}$. W.l.o.g. assume $f\left(t_{0}\right)>0$. Since $\left|f^{\prime}(t)\right| \leq \varepsilon$ a.e., use the lower bound $f\left(t_{0} \pm \tau\right) \geq$ $f\left(t_{0}\right)-\varepsilon \tau$ for $0 \leq \tau \leq \tau_{0}=f\left(t_{0}\right) / \varepsilon$ to obtain the estimate

$$
\int_{0}^{T} f^{2 k}(t) d t \geq \int_{t_{0}-\tau_{0}}^{t_{0}+\tau_{0}} f^{2 k}(t) d t \geq 2 \cdot \int_{0}^{\tau_{0}}(\varepsilon t)^{2 k} d t=\frac{2 \varepsilon^{2 k} \tau_{0}^{2 k+1}}{2 k+1}=\frac{2\|f\|_{\infty}^{2 k+1}}{(2 k+1) \varepsilon} .
$$

Proof of Theorem 3.2. Before going into technical details we lay out some heuristic ideas that motivate the strategy.

The presence of the definite terms in system (3.1) suggests using controls $u_{1}$ and $u_{2}$ with disjoint support. If one first keeps $u_{2} \equiv 0$, thus also $x_{4} \equiv 0$, and using a simple bang-bang control $u_{1}$ one easily reaches a point of the form $\left(0,0, q_{3}, 0,-q_{5}, 0\right)$ with $q_{3}, q_{5}>0$. Next one holds $u_{1} \equiv 0$ and, using a suitable control $u_{2}$, tries to reach a point $\left(0,0, q_{3}, q_{4},-q_{5},-q_{6}\right)$ with all $q_{i}>0$. But according to Lemma 3.6 this second step requires that the available time is sufficiently large (as determined by $\left.q_{3}\right)$. The subsequent estimates quantify this delicate trade-off, with the conclusion that, roughly speaking, if $x_{6}(T)<0$, then $\left|x_{5}(T)\right|<C T^{38 / 3}$ for all $T$ sufficiently small. The proof shows that this is true for all controls, not only for whatever clever combinations of the needle variations are used in the proof of Theorem 3.1. One of the key obstacles to making this heuristics into a general argument is that in such setting of all possible controls, there does not exist a clear notion of which of the two directions is obtained first. Thus the first objective is to identify an abstraction of "which of the directions $x_{5}$ and $x_{6}$ is generated first." Since

$\int_{0}^{T}\left(\int_{0}^{t} x_{1}^{2}(s) d s\right) x_{2}^{2}(t) d t+\int_{0}^{T} x_{1}^{2}(t)\left(\int_{0}^{t} x_{2}^{2}(s) d s\right) d t=\left(\int_{0}^{T} x_{1}^{2}(t) d t\right) \cdot\left(\int_{0}^{T} x_{2}^{2}(t) d t\right)$

and the system is invariant under the index permutation $\left(x_{1}, x_{2}, x_{3}, x_{4}, x_{5}, x_{6}\right) \mapsto$ $\left(x_{2}, x_{1}, x_{4}, x_{3}, x_{6}, x_{5}\right)$, we may assume w.l.o.g. that

$$
\int_{0}^{T}\left(\int_{0}^{t} x_{1}^{2}(s) d s\right) x_{2}^{2}(t) d t \geq \frac{1}{2}\left(\int_{0}^{T} x_{1}^{2}(t) d t\right)\left(\int_{0}^{T} x_{2}^{2}(t) d t\right),
$$

which may be rewritten in the most useful form

$$
\int_{0}^{T}\left(\int_{0}^{t} x_{1}^{2}(s) d s\right) x_{2}^{2}(t) d t \geq \frac{1}{2} \int_{0}^{T}\left(\int_{0}^{T} x_{1}^{2}(s) d s\right) x_{2}^{2}(t) d t .
$$

The key advantage of this form is that the weight against which $x_{2}^{2}$ is integrated is now a constant, so that Lemma 3.6 applies. If $x_{6}(T) \geq 0$, nothing needs to be shown. Thus assume $x_{6}(T)<0$. Then

$$
0>x_{6}(T)=\int_{0}^{T}\left(x_{3}^{2}(t) x_{2}^{2}(t)-x_{2}^{7}(t)\right) d t \geq \int_{0}^{T} x_{2}^{2}(t)\left(\frac{1}{2} \int_{0}^{T} x_{1}^{2}(s) d s-x_{2}^{5}(t)\right) d t
$$

and thus

$$
\xi_{2} \stackrel{\text { def }}{=} \max _{0 \leq t \leq T} x_{2}(t)>\left(\frac{1}{2} \int_{0}^{T} x_{1}^{2}(s) d s\right)^{\frac{1}{5}}
$$


The term on the right-hand side may be considered a cost, or energy, that is required to move $x_{6}(0)=0$ to $x_{6}(T)<0$. The next step is to find a lower bound for this energy in terms of the displacement. Using Lemma 3.7 with $\varepsilon=1$ and $f=x_{1}$ gives the crude estimate

$$
\int_{0}^{T} x_{1}^{2}(s) d s \geq 2 \cdot\left(\frac{1}{3}\right) \cdot \xi_{1}^{3} \stackrel{\text { def }}{=} \frac{2}{3} \max _{0 \leq t \leq T} x_{1}(t) .
$$

On the other hand, using Minkowski's inequality (backwards), we get

$$
-x_{5}(T)=\int_{0}^{T}\left(x_{1}^{7}(t)-x_{4}(t) x_{1}^{2}(t)\right) d t \leq \int_{0}^{T}\left|x_{1}^{7}(t)\right| d t \leq T \cdot \xi_{1}^{7} .
$$

Combining (3.18), (3.19), and (3.20) yields

$$
\xi_{2}>\left(\frac{1}{2} \int_{0}^{T} x_{1}^{2}(s) d s\right)^{\frac{1}{5}} \geq\left(\frac{1}{2} \cdot \frac{2}{3} \xi_{1}^{3}\right)^{\frac{1}{5}} \geq\left(\frac{1}{3}\left(\frac{-x_{5}(T)}{T}\right)^{\frac{3}{7}}\right)^{\frac{1}{5}} .
$$

Using that $\xi_{2} \leq \frac{1}{2} T\left(\right.$ from $u_{2}(\cdot) \mid \leq 1$ together with $\left.x_{2}(0)=x_{2}(T)=0\right)$ implies

$$
-x_{5}(T) \leq 3^{\frac{1}{5}} \cdot \xi_{2}^{\frac{35}{3}} \cdot T \leq 3^{\frac{1}{5}} \cdot 2^{-\frac{35}{3}} \cdot T^{\frac{38}{3}} .
$$

Note that, in particular, if $x(T)=(0,0, *, *,-\rho,-\rho) \in \mathcal{R}_{0}(T)$ with $\rho>0$, then $\rho<C T^{38 / 3}$ (with $C=3^{1 / 5} \cdot 2^{-35 / 3}$ ).

THEOREM 3.8. There exists a constant $C>0$ such that for all sufficiently small $T>0$ the image $\varphi(\mathcal{R}(T))$ of the reachable set of system (3.1) contains the open ball $B_{0}^{\infty}\left(C T^{40 / 3}\right)$.

Corollary 3.9. The system (3.1) is STLOC about $y=0$.

COROLLARY 3.10. The approximating cone $\overline{\mathcal{K}}_{\varphi}^{\frac{40}{3}}$ is the whole tangent space $T_{0} \mathbb{R}^{4}$.

Main elements of the construction. These statements all follow directly from Theorem 4.3 and we refer to its proof for the full technical details. At this point we illustrate the critical elements in the construction showing a slightly weaker result. The general proof given in section 4 requires no major innovation but mainly involves keeping track of many more quantities.

The objective in this construction is to suitably combine the families of control variations

$$
u_{s}^{(1)}(t)=\left\{\begin{array}{ll}
(+1,0) & \text { if } 0 \leq t<\frac{s}{2}, \\
(-1,0) & \text { if } \frac{s}{2} \leq t<s
\end{array} \text { and } u_{s}^{(2)}(t)= \begin{cases}(0,+1) & \text { if } 0 \leq t<\frac{s}{2} \\
(0,-1) & \text { if } \frac{s}{2} \leq t<s\end{cases}\right.
$$

which steer to points $y\left(s, u_{s}^{(1)}\right)=\left(0,0,-2^{-7} s^{8}, 0\right)$ and $y\left(s, u_{s}^{(2)}\right)=\left(0,0,0,-2^{-7} s^{8}\right)$ on the negative $y_{3}$ and $y_{4}$ axes to obtain families of control variations that steer to points in the third quadrant $y_{3}<0$ and $y_{4}<0$ in the plane $y_{1}=y_{2}=0$. Thus consider the control

$$
u(t)= \begin{cases}(+1,0) & \text { if } \quad 0 \leq t<t_{1}=c \varepsilon^{r} \\ (-1,0) & \text { if } \quad t_{1} \leq t<t_{2}=2 c \varepsilon^{r} \\ (0,+1) & \text { if } \quad t_{2} \leq t<t_{3}=2 c \varepsilon^{r}+\varepsilon+\mu \varepsilon^{m}, \\ (0,-1) & \text { if } \quad t_{3} \leq t<T=2 c \varepsilon^{r}+2 \varepsilon+2 \mu \varepsilon^{m}\end{cases}
$$

with $r, m \geq 1$ and constants $c, \mu$ to be determined (first think of $r=1$ and $\mu=0$ ). 
Verify that at selected switching times the trajectory passes through

$$
\begin{aligned}
& x\left(t_{1}\right)=\left(c \varepsilon^{r}, 0, \frac{1}{3} c^{3} \varepsilon^{3 r}, 0,-\frac{1}{8} c^{8} \varepsilon^{8 r}, 0\right), \\
& x\left(t_{2}\right)=\left(0,0, \frac{2}{3} c^{3} \varepsilon^{3 r}, 0,-\frac{1}{4} c^{8} \varepsilon^{8 r}, 0\right), \\
& x\left(t_{3}\right)=\left(0, \varepsilon+\mu \varepsilon^{m}, \frac{2}{3} c^{3} \varepsilon^{3 r}, \frac{1}{3}\left(\varepsilon+\mu \varepsilon^{m}\right)^{3},-\frac{1}{4} c^{8} \varepsilon^{8 r} \frac{2}{9} c^{3} \varepsilon^{3+3 r}-\frac{1}{8} \varepsilon^{8}+p_{36} \cdot \mu \varepsilon^{m}\right), \\
& x(T)=\left(0,0, \frac{2}{3} c^{3} \varepsilon^{3 r}, \frac{2}{3}\left(\varepsilon+\mu \varepsilon^{m}\right)^{3},-\frac{1}{4} c^{8} \varepsilon^{8 r}, \frac{4}{9} c^{3} \varepsilon^{3+3 r}-\frac{1}{4} \varepsilon^{8}+p_{46} \cdot \mu \varepsilon^{m}\right) .
\end{aligned}
$$

Here $p_{36}$ and $p_{46}$ are polynomial expressions in $\left(c, \varepsilon, \varepsilon^{r}, \mu \varepsilon^{m}\right)$. In particular,

$$
p_{46}=\left(\frac{4}{3} c^{3} \varepsilon^{3 r+2}-2 \varepsilon^{7}\right)+q_{46} \cdot \mu \varepsilon^{m},
$$

where $q_{46}$ is a polynomial expression in $\left(c, \varepsilon, \varepsilon^{r}, \mu \varepsilon^{m}\right)$. First consider the case with $\mu=0$ (no perturbation). If $r=1$, then $x_{6}\left(2 c \varepsilon^{r}+2 \varepsilon\right)>0$ for all sufficiently small $\varepsilon>0$. However, (still with $\mu=0$ ) if one chooses $r \geq \frac{5}{3}$, then one may obtain $y_{4}(T)<0$ for suitable choices of $c$. Choosing the critical value $r=\frac{5}{3}$ yields equality of the exponents in $\varepsilon^{8}$ and $\varepsilon^{3+3 r}$, and thus by varying the parameter $c$ one reaches the points $y(T)=\varepsilon^{\frac{40}{3}}\left(0,0,-\frac{1}{4} c^{8}, \frac{4}{9} c^{3}-\frac{1}{4}\right)$ in the third quadrant of the plane $y_{1}=y_{2}=0$.

Alternatively, fix the value $c^{*}=(9 / 16)^{1 / 3}$, which yields $y_{4}(T)=0$ when $\mu=0$. To reach a given point $\eta=\left(0,0, \eta_{3}, \eta_{4}\right)$ with $\eta_{3}, \eta_{4}<0$ first choose $\varepsilon=(16 / 9)^{1 / 5}$. $\left(-4 \eta_{3}\right)^{3 / 40}$. Then, using that $\left.p_{46}\right|_{\mu=0} \neq 0$, the implicit function theorem guarantees that (for $-\eta_{4}>0$ sufficiently small) one can solve $\eta_{4}=\mu \varepsilon^{m} \cdot p_{46}\left(c, \varepsilon, \varepsilon^{r}, \mu \varepsilon^{m}\right.$ ) (with $m=\frac{40}{3}$ ) for $\mu>0$.

Note that the total time $T=2 \varepsilon+2 c \varepsilon^{\frac{5}{3}}+2 \mu \varepsilon^{\frac{40}{3}} \stackrel{!}{=} s$ is of order $\varepsilon($ for $\varepsilon<1)$, and it is straightforward to reparameterize the controls choosing a constant multiple $s=a \varepsilon$ so that they qualify as a family of control variations at zero. Such linear reparameterization clearly does not affect the exponents such as $\frac{40}{3}$, thus yielding the statements in Theorem 3.8 and its corollaries. The construction of controls steering to any point $\eta \in \mathbb{R}^{4}$ with $\eta_{1}, \eta_{2}$ not necessarily zero requires no major innovation, just much book-keeping, and it is completely analogous to the proof of Theorem 4.3 given below.

Remark 3.11. Recall that one commonly linearly reparameterizes needle variations when constructing convex combinations of tangent vectors as tangent vectors of the same order. What is new here is that one must consider nonlinear reparameterizations (here basically replace $s$ by a power $s^{r}$ with $r$ strictly larger than one). As a consequence one may still obtain the convex combinations of the original tangent vectors here but not as tangent vectors of the same order. (Here the combination of eighth order tangent vectors yields at best tangent vectors of order (40/3) as shown above.) Further, much more severe implications for controllability properties in general are analyzed in section 5 .

In summary - using the traditional language of STLC - the analysis of system (3.1) quantified the delicate interplay of simultaneous neutralization of two obstructions to controllability by (what initially might appear to be) higher order terms: The terms $\int x_{4} x_{1}^{2}$ and $\int x_{3} x_{2}^{2}$, which are always nonnegative, are 6th order in time, whereas the indefinite terms $\int x_{2}^{7}$ and $\int x_{1}^{7}$ are 8 th order in time. The constructions in this section showed that it is indeed possible to reach points $p=\left(p_{1}, \ldots, p_{6}\right)$ with either $p_{5}<0$ or $p_{6}<0$ from $x(0)=0$ in a time of order $\|p\|_{\infty}^{1 / 8}$, and even points $p$ with both $p_{5}<0$ and $p_{6}<0$, but only in time of order at least $\|p\|_{\infty}^{3 / 38}$ - as a result we have the nonconvex approximating cones for $8 \leq m<\frac{40}{3}$ sketched in Figure 3.1. The critical value obtained in this section is the exponent $\frac{5}{3}>1$, which relates the maximal distance the variation can be translated to the length of the needle variation. 
These phenomena never played a role in the classical study STLC as the lower order obstructions $\int x_{1}^{2}$ and $\int x_{2}^{2}$ cannot be neutralized, making any further study of the system in view of STLC irrelevant. Nonetheless, not only does this example demonstrate a noteworthy feature from the point of view of STLOC, but it also serves as the foundation of constructions in the next sections, which basically replace the role of the output map $\varphi: x \mapsto\left(x_{1}, x_{2}, x_{5}, x_{6}\right)$ by a nonstationary reference trajectory in order to obtain unexpected controllability properties.

4. A nonstationary reference trajectory. A slight modification of this system with output yields a corresponding result for controllability about (and optimality of) a nonstationary reference trajectory. It might well be possible to construct a similar example with fewer controls. We chose this implementation with four controls for its structural simplicity and because it allows one to easily build on the results of the previous section.

$$
\left\{\begin{array}{lrl}
\dot{x}_{1} & =u_{1}, & \left|u_{1}(\cdot)\right| \leq 1, \\
\dot{x}_{2}=u_{2}, & \left|u_{2}(\cdot)\right| \leq 1, \\
\dot{x}_{3}=x_{1}^{2}+\left(1+u_{01}\right), & \left|u_{01}(\cdot)\right| \leq 1, \\
\dot{x}_{4}=x_{2}^{2}+\left(1+u_{02}\right), & \left|u_{02}(\cdot)\right| \leq 1, \\
\dot{x}_{5}=x_{4} x_{1}^{2}-x_{1}^{7}, & x(0)=0, \\
\dot{x}_{6}=x_{3} x_{2}^{2}-x_{2}^{7}, & x^{*}(t)=(0,0, t, t, 0,0) .
\end{array}\right.
$$

The key in this construction is that the lower order (i.e., apparently dominant) definite components $\int_{0}^{T} x_{1}^{2}(t) d t$ and $\int_{0}^{T} x_{2}^{2}(t) d t$ are aligned with the direction of the nonstationary reference trajectory and that the zero speed $\|\dot{x}\|=0$ is on the boundary of the set of admissible velocities (on the hyperplane $x_{1}=x_{2}=0$, which includes the reference trajectory). With the fixed boundary velocity $u_{01}=u_{02}=-1$ the system may be considered a two-input system (with controls $\left(u_{1}, u_{2}\right)$ ) about the stationary reference trajectory $x \equiv 0$ (basically system (3.1)). This two-input system is not STLC as obviously the $\left(x_{3}, x_{4}\right)$-directions are uncontrollable. (It is impossible to reach points $x$ with $x_{3}<0$ or $x_{4}<0$ from $x=0$.) But in (4.1) these uncontrollable directions are aligned with (as opposed to transversal to) the nonstationary reference trajectory, and thus controllability involves comparison with $\dot{x}_{3}=\dot{x}_{4} \equiv 1$ (as opposed to $\dot{x}_{3}=\dot{x}_{4} \equiv 0$ ).

With this design in mind, it is natural to combine standard techniques and results about STLC and convexity of approximating cones that apply to systems about an equilibrium point (i.e., first holding $u_{01}=u_{02}=-1$ fixed) with constructions unique to nonstationary reference trajectories. In particular, after having generated desired tangent vectors working in the vicinity of the equilibrium point, it is straightforward to catch up with the prescribed nonstationary reference trajectory by using $u_{01}>0$ and $u_{02}>0$; compare Figure 4.1 for a schematic illustration.

THEOREM 4.1. For $8 \leq m<\frac{38}{3}$ the approximating cones $\overline{\mathcal{K}}_{0}^{m}$ of system (4.1) are not convex as they have inward corners in the sense that

$$
\left(0,0,0,0, v_{5}, v_{6}\right) \in \overline{\mathcal{K}}_{0}^{m} \Longleftrightarrow\left(v_{5} \geq 0 \text { or } v_{6} \geq 0\right) .
$$

Proof. First note that if $\xi(\cdot, u)$ and $x(\cdot, u)$ denote the solution curves of systems (3.1) and (4.1), respectively (for the same control), then clearly $x_{5}(t, u) \geq \xi_{5}(t, u)$ and $x_{6}(t, u) \geq \xi_{6}(t, u)$ for all $t \geq 0$. Thus, it is a direct consequence of Theorem 3.2 that if $m<\frac{38}{3}, v_{5}<0$, and $v_{6}<0$, then $\left(0,0,0,0, v_{5}, v_{6}\right) \notin \mathcal{K}_{0}^{m}$.

In the other direction, we show that every $v=\left(0,0, v_{3}, v_{4}, v_{5}, v_{6}\right) \in \mathbb{R}^{6}$ with $v_{5} \geq 0$ or $v_{6} \geq 0$ is contained in the cones $\overline{\mathcal{K}}_{0}^{m}$ for $m \geq 8$. (More general conclusions 


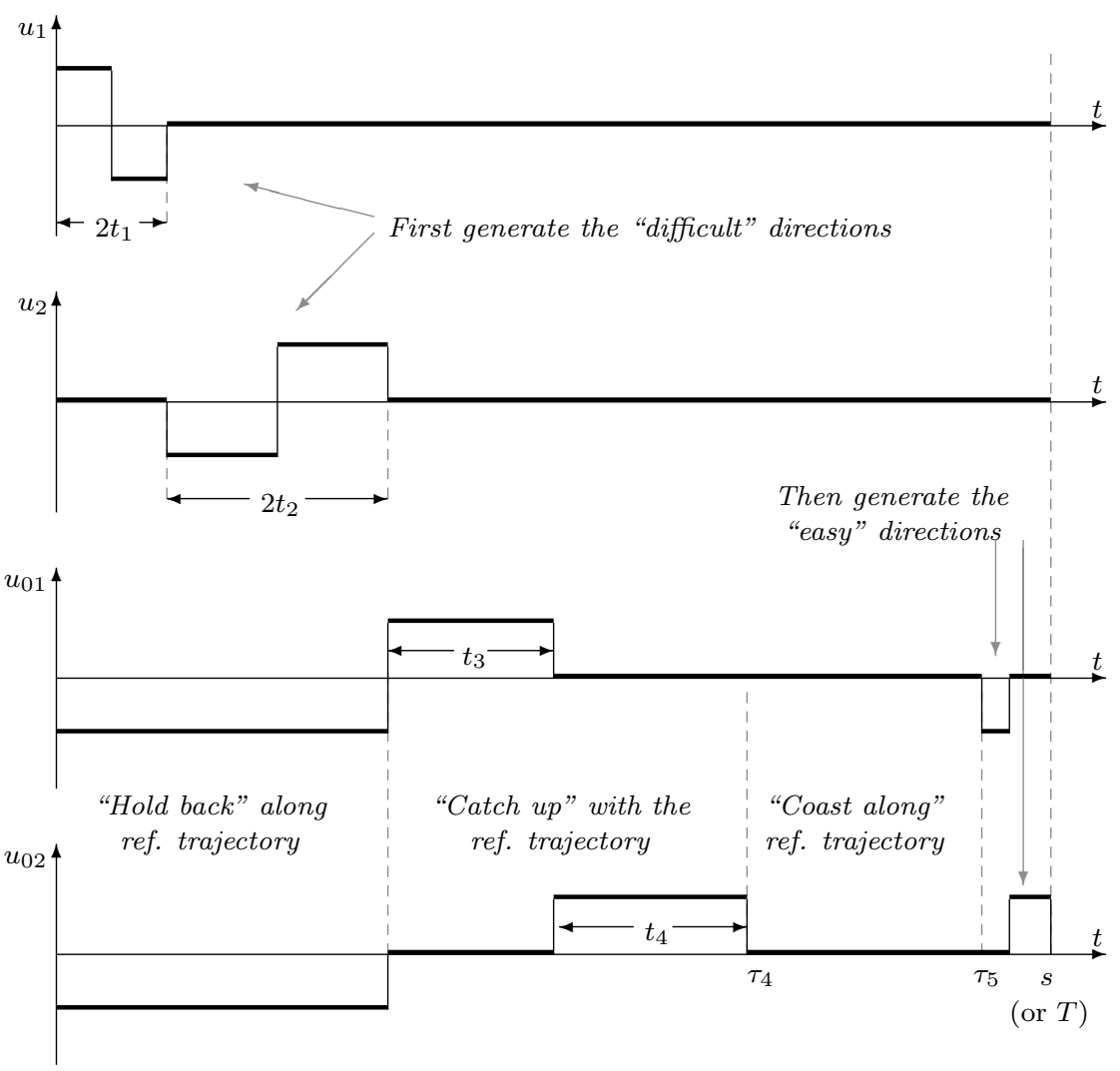

FIG. 4.1. Schematic design of the needle variations for the proof of Theorem 4.1.

allowing $v_{1}, v_{2} \neq 0$ may be obtained using Theorem 4.3.) Suppose $0<s<1$ is given and $q=\left(0,0, q_{3}, q_{4}, q_{5}, q_{6}\right) \in R^{6}$ is such that $\|q\|_{\infty}<(1 / 26)^{8}$ and w.l.o.g. $q_{6} \geq 0$. We exhibit a family of control variations at zero, $u_{s}=u_{s, q}:[0, s] \mapsto[-1,1]^{4}, 0 \leq s \leq 1$, such that $x\left(s, u_{s}\right)=x^{*}(s)+s^{8} q$. Write $t_{1}=s\left(4\left|q_{5}\right|\right)^{1 / 8}$, and let $t_{2} \geq 0$ be the unique nonnegative number such that $s^{8} q_{6}=\frac{2}{9} t_{1}^{3} t_{2}^{3}+\frac{1}{8} t_{2}^{8}$. Moreover, let $t_{3}=2 t_{1}+2 t_{2}-\frac{2}{3} t_{1}^{3}$, and $t_{4}=2 t_{1}+2 t_{2}-\frac{2}{3} t_{2}^{3}$. Write $\tau_{4}=2 t_{1}+2 t_{2}+t_{3}+t_{4}$ and $\tau_{5}=s-s^{8}\left(\left|q_{3}\right|+\left|q_{4}\right|\right)$.

It is critical that $\tau_{4} \leq \tau_{5}$. To see this, note that $t_{i} \leq 2 s\|q\|_{\infty}^{1 / 8}$ for both $i=1,2$. Next $t_{i} \leq 8 s\|q\|_{\infty}^{1 / 8}$ for both $i=3,4$, and thus $\tau_{4} \leq 24 s\|q\|_{\infty}^{1 / 8}$. On the other hand, $\tau_{5} \geq s-2 s\|q\|_{\infty}^{1 / 8}$ (since both $s<1$ and $\|q\|_{\infty}<1$ ). Thus $\tau_{5}-\tau_{4} \geq s\left(1-26\|q\|_{\infty}^{1 / 8}\right) \geq 0$ follows from the assumption that $\|q\|_{\infty}<(1 / 26)^{8}$. Consequently, the following is a well-defined one-parameter family of control variations of $u^{*} \equiv(0,0,0,0)$ :

$$
u(t)=\left\{\begin{array}{ccc}
\left(\operatorname{sign}\left(q_{5}\right), 0,-1,-1\right) & \text { if } & 0 \leq t<t_{1}, \\
\left(-\operatorname{sign}\left(q_{5}\right), 0,-1,-1\right) & \text { if } & t_{1} \leq t<2 t_{1}, \\
(0,1,-1,-1) & \text { if } & 2 t_{1} \leq t<2 t_{1}+t_{2}, \\
(0,-1,-1,-1) & \text { if } & 2 t_{1}+t_{2} \leq t<2 t_{1}+2 t_{2}, \\
(0,0,1,0) & \text { if } & 2 t_{1}+2 t_{2} \leq t<2 t_{1}+2 t_{2}+t_{3}, \\
(0,0,0,1) & \text { if } & 2 t_{1}+2 t_{2}+t_{3} \leq t<\tau_{4}, \\
(0,0,0,0) & \text { if } & \tau_{4} \leq t<\tau_{5}, \\
\left(0,0, \operatorname{sign}\left(q_{3}\right), 0\right) & \text { if } & \tau_{5} \leq t<s-s^{8}\left|q_{4}\right|, \\
\left(0,0,0, \operatorname{sign}\left(q_{4}\right)\right) & \text { if } & s-s^{8}\left|q_{4}\right| \leq t<s .
\end{array}\right.
$$


Note that as a family of control variations at zero this is a family of needle variations. One easily verifies that at selected switching times the trajectory passes through

$$
\begin{aligned}
x\left(2 t_{1}\right) & =\left(0,0, \frac{2}{3} t_{1}^{3}, 0, s^{8} q_{5}, 0\right), \\
x\left(2 t_{1}+2 t_{2}\right) & =\left(0,0, \frac{2}{3} t_{1}^{3}, \frac{2}{3} t_{2}^{3}, s^{8} q_{5}, s^{8} q_{6}\right), \\
x\left(\tau_{4}\right) & =\left(0,0, \tau_{4}, \tau_{4}, s^{8} q_{5}, s^{8} q_{6}\right), \\
x\left(\tau_{5}\right) & =\left(0,0, \tau_{5}, \tau_{5}, s^{8} q_{5}, s^{8} q_{6}\right), \\
x(s) & =(0,0, s, s, 0,0)+s^{8} q .
\end{aligned}
$$

The flow $\Phi$ corresponding to the control $u^{*} \equiv 0$ satisfies $\Phi_{-s}\left(0,0, x_{3}, x_{4}, x_{5}, x_{6}\right)=$ $\left(0,0, x_{3}-s, x_{4}-s, x_{5}, x_{6}\right)$, and thus $x\left(s, u_{s}\right)=x\left(s, u^{*}\right)+s^{8} \Phi_{s}\left(0,0, q_{3}, q_{4}, q_{5}, q_{6}\right)$. Consequently $\left(0,0, \lambda q_{3}, \lambda q_{4}, \lambda q_{5}, \lambda q_{6}\right) \in \overline{\mathcal{K}}_{0}^{8}$ for all $\lambda \geq 0$.

The proof showed, in particular, that the vectors $(0,0,0,0,-1,0)$ and $(0,0,0,0,0$, $-1)$ are members of $\overline{\mathcal{K}}_{0}^{8}$ that are generated by needle variations. In contrast, according to Theorem 3.2, the convex combination $\frac{1}{2}(0,0,0,0,-1,-1)$ cannot at all be generated as an eighth order tangent vector. In particular, it cannot be generated by any (however clever) combination of needle variations. Nonetheless, in analogy to Corollary 3.10, in this particular system, this convex combination can still be generated as an even higher order tangent vector as shown below. First we establish a basic estimate needed later.

Lemma 4.2. If $0<a<M^{5}$ and $0<b<M^{8}$, then the unique positive root $t_{0}$ of the scalar function $f(t)=b+a t^{3}-\frac{1}{8} t^{8}$ satisfies $t_{0}<2 M$.

Proof. The existence of a unique positive root is elementary. Set $t_{00}=(8 a)^{1 / 5}$, which is the first positive root of $t \mapsto a t^{3}-\frac{1}{8} t^{8}$. Since $f^{\prime \prime}(t)<0$ for all $t \geq t_{00}$, the linear estimate $0 \stackrel{!}{=} f\left(t_{0}\right) \leq f\left(t_{00}\right)+f^{\prime}\left(t_{00}\right)\left(t_{0}-t_{00}\right)=b-5 \cdot 8^{2 / 5} a^{7 / 5}\left(t_{0}-(8 a)^{1 / 5}\right)$ yields the bound

$$
t_{00} \leq t_{0} \leq t_{00}+\frac{b}{10 \cdot 2^{1 / 5} a^{7 / 5}}=\frac{10 \cdot 2^{4 / 5} a^{8 / 5}+b}{10 \cdot 2^{1 / 5} a^{7 / 5}} .
$$

In the case that $b<a^{8 / 5}$, this immediately yields the desired estimate $t_{0}<2 a^{1 / 5} \leq$ $2 M$. Alternatively, if $a$ is too small, i.e., if $b \geq a^{8 / 5}$, we find directly that $f\left(2 b^{1 / 8}\right) \leq$ $b+b^{5 / 8}\left(2 b^{1 / 8}\right)^{3}-\frac{1}{8}\left(2 b^{1 / 8}\right)^{8}=-23 b<0$, and hence the first positive zero $t_{0}$ of $f$ again satisfies $x_{0} \leq 2 b^{1 / 8}<2 M$.

THEOREM 4.3. For every $T>0$ sufficiently small, the reachable set $\mathcal{R}(T)$ of system (4.1) contains the open ball $B_{x^{*}(T)}^{\infty}\left(\left(2^{-5} T\right)^{40 / 3}\right)$ centered at $x^{*}(T)$.

COROLLARY 4.4. The system (4.1) is $S T L C$ about the reference trajectory $x^{*}(t)=$ $(0,0, t, t, 0,0)$.

COROLlary 4.5. The approximating cone $\overline{\mathcal{K}}_{0}^{40 / 3}$ of system (4.1) is the whole tangent space $\overline{\mathcal{K}}_{0}^{40 / 3}=T_{0} \mathbb{R}^{6}$.

Proof. Similar to the proof of Theorem 4.1 we explicitly construct for any given time $T>0$ and any given point $q$ sufficiently close to $x^{*}(T)$ a control $u=u_{q}:[0, T] \mapsto$ $[-1,1]^{4}$ which steers the system to $q$. The main differences to the prior work is that now we need to also consider the case when $q_{1} \neq 0$ and $q_{2} \neq 0$. Note that since suitable open mapping principles for approximating cones for this setting are not available, we cannot rely on just generating tangent vectors.

Suppose $0<T<1$ and $q \in B_{0}^{\infty}\left(2^{-40} T^{40 / 3}\right)$ are given. We consider only the case that both $q_{5}<0$ and $q_{6}<0$. All other cases can be handled analogously, albeit 
with considerably simpler constructions. The basic construction for moving into the difficult directions $(0,0,0,0, *, *)$ is the same as in earlier proofs. These are followed by simple control actions that move into the easy directions. However, these later moves also have some effect on the difficult directions. Thus we first calculate these side effects and modify the initial controls to overshoot the targets in the difficult directions. The required corrections are calculated to cancel the integrals that result from (4.11) (also compare (4.12)).

$$
\begin{gathered}
\Delta q_{31}=q_{1}^{2}\left|q_{2}\right|=\int_{0}^{\left|q_{2}\right|} q_{1}^{2} d t, \quad \Delta q_{3}=\frac{1}{3}\left|q_{1}\right|^{3}+\left|q_{2}\right| q_{1}^{2}=\Delta q_{31}+\int_{0}^{\left|q_{1}\right|}\left(\sigma_{1} t\right)^{2} d t \\
\Delta q_{4}=\frac{1}{3}\left|q_{2}\right|^{3}=\int_{0}^{\left|q_{2}\right|} t^{2} d t \\
\Delta q_{51}=q_{1}^{2}\left|q_{2}\right|\left(T+q_{4}\right)-\frac{1}{2} q_{1}^{2} q_{2}^{2}-\frac{1}{4} q_{1}^{2} q_{2}^{4}-q_{1}^{7}\left|q_{2}\right| \\
=\int_{0}^{\left|q_{2}\right|}\left(\left(\tau_{8}+q_{4}-\Delta q_{4}+\left(t+\frac{1}{3} t^{3}\right)\right) q_{1}^{2}-q_{1}^{7}\right) d t \\
\Delta q_{5}=\Delta q_{51}+\frac{1}{3}\left|q_{1}\right|^{3}\left(T+q_{4}\right)-\frac{1}{3}\left|q_{1}\right|^{3}\left|q_{2}\right|-\frac{1}{9}\left|q_{1}\right|^{3}\left|q_{2}\right|^{3}-\frac{1}{12} q_{1}^{4}-\frac{1}{8}\left|q_{1}\right| q_{1}^{7} \\
=\Delta q_{51}+\int_{0}^{\left|q_{1}\right|}\left(\left(\tau_{7}+t+q_{4}-\Delta q_{4}\right)\left(\sigma_{1} t\right)^{2}+\left(\sigma_{1} t\right)^{7}\right) d t \\
\Delta q_{6}=\frac{1}{3}\left|q_{2}\right|^{3} T+\frac{1}{3}\left|q_{2}\right|^{3} q_{3}-\frac{1}{12} q_{1}^{2} q_{2}^{4}-\frac{1}{12} q_{2}^{4}-\frac{1}{8}\left|q_{2}\right| q_{2}^{7} \\
=\int_{0}^{\left|q_{2}\right|}\left(\left(\tau_{8}+q_{3}-\Delta q_{31}+\left(q_{1}^{2}+1\right) t\right)\left(\sigma_{2} t\right)^{2}-\left(\sigma_{2} t\right)^{7}\right) d t
\end{gathered}
$$

What really matters in the subsequent construction is the size of these correction terms - clearly they are all of order at least 3 in $\|q\|_{\infty}$. Next let $t_{1}=\left(-4\left(q_{5}-\Delta q_{5}\right)\right)^{\frac{1}{8}}$, $t_{2} \geq 0$, be the smallest nonnegative number such that $\left(q_{6}-\Delta q_{6}\right)=\frac{2}{9} t_{1}^{3} t_{2}^{3}-\frac{1}{8} t_{2}^{8}$ (which is unique in the challenging case of $\left.\left(q_{6}-\Delta q_{6}\right)<0\right)$. Let $t_{3}=2 t_{1}+2 t_{2}-\frac{2}{3} t_{1}^{3}$ and $t_{4}=2 t_{1}+2 t_{2}-\frac{2}{3} t_{2}^{3}$. Write $\tau_{3}=2 t_{1}+2 t_{2}+t_{3}, \tau_{4}=2 t_{1}+2 t_{2}+t_{3}+t_{4}$ and working backwards $\tau_{8}=T-\left|q_{2}\right|, \tau_{7}=\tau_{8}-\left|q_{1}\right|, \tau_{6}=\tau_{7}-\left|q_{4}-\Delta q_{4}\right|, \tau_{5}=\tau_{6}-\left|q_{3}-\Delta q_{3}\right|$. Also use the abbreviations $\sigma_{i}=\operatorname{sign}\left(q_{i}\right)$ for $i=1,2$ and $\sigma_{i}=\operatorname{sign}\left(q_{i}-\Delta q_{i}\right)$ for $i=3,4$. Define the control $u=u_{q, T}:[0, T] \mapsto[-1,1]^{4}$ by

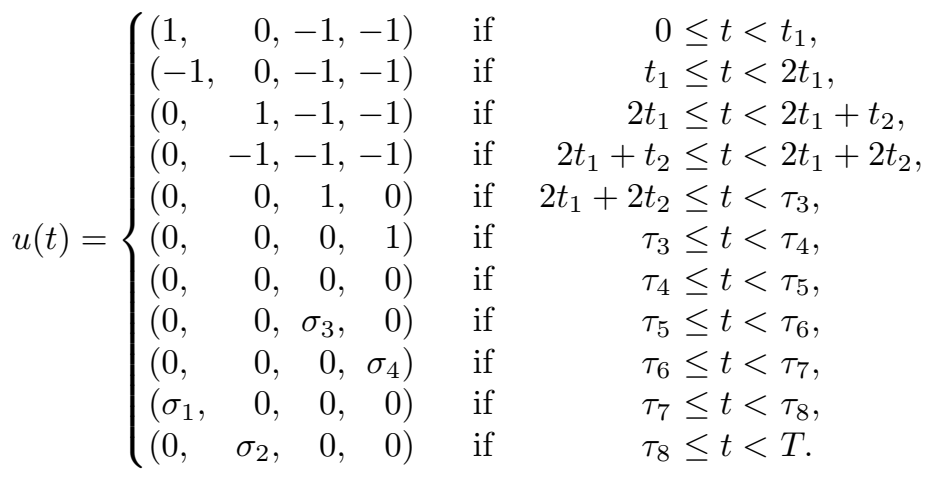


It is essential to verify that $\tau_{4} \leq \tau_{5}$. Start with the crude estimate $\left|\Delta q_{i}\right| \leq 2\|q\|_{\infty}^{3} \leq$ $2\|q\|_{\infty}$ for $i=1,2,5,6$. Next $t_{1} \leq 2\|q\|_{\infty}^{1 / 8}$. The critical estimate is for $t_{2}$-note the sign reversal in the defining equation in this construction compared to the one in the proof of Theorem 4.1: The earlier construction allowed one to estimate $s^{8} q_{6}=\frac{2}{9} t_{1}^{3} t_{2}^{3}+$ $\frac{1}{8} t_{2}^{8} \stackrel{!}{\geq} \frac{1}{8} t_{2}^{8}$ yielding $t_{2} \leq\left(8 s^{8} q_{-} 6\right)^{1 / 8}$. Now in the interesting case $0 \geq\left(q_{6}-\Delta q_{6}\right)=$ $\frac{2}{9} t_{1}^{3} t_{2}^{3}-\frac{1}{8} t_{2}^{8}$ Lemma 4.2 applies with $a=\frac{2}{9} t_{1}^{3} \leq \frac{4}{9}\|q\|_{\infty}^{3 / 8}, b=\left(\Delta q_{6}-q_{6}\right) \leq 3\|q\|_{\infty}$, and $M=\|q\|_{\infty}^{3 / 40}$, yielding $t_{2} \leq 2\|q\|_{\infty}^{3 / 40}$. Easily $t_{i} \leq 8\|q\|_{\infty}^{3 / 40}$ for $i=3$, 4 , and thus $\tau_{4} \leq 24\|q\|_{\infty}^{3 / 40}$.

On the other hand, $T-\tau_{5} \leq 4\|q\|_{\infty}+\left|\Delta q_{5}\right|+\left|\Delta q_{6}\right| \leq 8\|q\|_{\infty} \leq 8\|q\|_{\infty}^{3 / 40}$. Together with $0<T<1$ and $\|q\|_{\infty}<\left(2^{-5} T\right)^{\frac{40}{3}}$ this yields $\tau_{5}-\tau_{4} \geq T-32\|q\|_{\infty}^{3 / 40} \geq 0$.

Note that $x_{1}(t)=x_{2}(t)=0$ for all $t \in\left[2 t_{1}+2 t_{2}, \tau_{7}\right]$. Thus $x_{5}(\cdot)$ and $x_{6}(\cdot)$ are constant on this interval. It is straightforward to verify that at selected switching times the trajectory passes through

$$
\begin{aligned}
x\left(t_{1}\right) & =\left(t_{1}, 0, \frac{1}{3} t_{1}^{3}, 0,-\frac{1}{8} t_{1}^{8}, 0\right), \\
x\left(2 t_{1}\right) & =\left(0,0, \frac{2}{3} t_{1}^{3}, 0, q_{5}-\Delta q_{5}, 0\right), \\
x\left(2 t_{1}+t_{2}\right) & =\left(0, t_{2}, \frac{2}{3} t_{1}^{3}, \frac{1}{3} t_{2}^{3}, q_{5}-\Delta q_{5},-\frac{1}{8} t_{2}^{8}+\frac{2}{9} t_{1}^{3} t_{2}^{3}\right), \\
x\left(2 t_{1}+2 t_{2}\right) & =\left(0,0, \frac{2}{3} t_{1}^{3}, \frac{2}{3} t_{2}^{3}, q_{5}-\Delta q_{5}, q_{6}-\Delta q_{6}\right), \\
x\left(\tau_{3}\right) & =\left(0,0, \tau_{3}, t_{3}+\frac{2}{3} t_{2}^{3}, q_{5}-\Delta q_{5}, q_{6}-\Delta q_{6}\right), \\
x\left(\tau_{4}\right) & =\left(0,0, \tau_{4}, \tau_{4}, q_{5}-\Delta q_{5}, q_{6}-\Delta q_{6}\right), \\
x\left(\tau_{5}\right) & =\left(0,0, \tau_{5}, \tau_{5}, q_{5}-\Delta q_{5}, q_{6}-\Delta q_{6}\right), \\
x\left(\tau_{6}\right) & =\left(0,0, \tau_{6}+q_{3}-\Delta q_{3}, \tau_{6}, q_{5}-\Delta q_{5}, q_{6}-\Delta q_{6}\right), \\
x\left(\tau_{7}\right) & =\left(0,0, \tau_{7}+q_{3}-\Delta q_{3}, \tau_{7}+q_{4}-\Delta q_{4}, q_{5}-\Delta q_{5}, q_{6}-\Delta q_{6}\right), \\
x\left(\tau_{8}\right) & =\left(q_{1}, 0, \tau_{8}+q_{3}-\Delta q_{31}, \tau_{8}+q_{4}-\Delta q_{4}, q_{5}-\Delta q_{51}, q_{6}-\Delta q_{6}\right), \\
x(T) & =\left(q_{1}, q_{2}, T+q_{3}, T+q_{4}, q_{5}, q_{6}\right) .
\end{aligned}
$$

Note that the construction above was given for a fixed terminal time $T$. But it is easily modified to yield families of control variations $u_{s}:[0, s] \mapsto U$ at zero by replacing $T$ by $s$ and $q$ by $s^{40 / 3} q$. This yields the curve of endpoints $x\left(s, u_{s}\right)=(0,0, s, s, 0,0)+s^{40 / 3} q$ yielding $q \in \overline{\mathcal{K}}_{0}^{40 / 3}$.

In summary, this section modified the system (3.1) so that its STLOC properties translate into even less expected properties of STLC about a nonstationary reference trajectory. The key step in the construction is to align the obstructions $\int x_{1}^{2}$ and $\int x_{2}^{2}$ with the reference trajectory and to utilize control values that lie on the boundary of the control set $U$. As a result, the difficult directions (negative in $x_{5}$ and negative in $x_{6}$ ) can only be generated by needle variations at $t=0$, which moreover can only be moved by time intervals whose length is a power of the length of the variation that is strictly larger than 1 (here $5 / 3$ ). The tangent cones again exhibit loss of convexity for $8 \leq m<\frac{38}{3}$, as illustrated in Figure 3.1.

5. Delicate structural instability. This section analyzes small modifications of the systems considered in the preceding sections. The main result is that what at first appear to be "at a higher order" perturbations can nonetheless destroy the delicate controllability exhibited in the earlier sections. More specifically, we exhibit a polynomial cascade system which has nonconvex approximating cones generated by needle variations and which is not controllable. 
Returning to the discussion of the system (4.1), we recall that cones $\overline{\mathcal{K}}_{0}^{8}$ are not convex. While the vectors $(0,0,0,0,-1,0) \in \overline{\mathcal{K}}_{0}^{8}$ and $(0,0,0,0,0,-1) \in \overline{\mathcal{K}}_{0}^{8}$ could be generated by needle variations at zero as 8 th order tangent vectors, it was shown that it is impossible to obtain convex combinations of these as tangent vectors of the same order. However, such convex combinations can be generated as even higher order tangent vectors, e.g., $\left(0,0,0,0,-\frac{1}{2},-\frac{1}{2}\right) \in \overline{\mathcal{K}}_{0}^{40 / 3}$ by fairly delicate constructions. As we illustrate next, such constructions can be rendered impossible through quite innocent-looking perturbations. Similar to section 3, we start again with an affine system with output and a stationary reference trajectory:

$$
\left\{\begin{array}{lr}
\dot{z}_{1}=u_{1}, & \left|u_{1}(\cdot)\right| \leq 1, \\
\dot{z}_{2}=u_{2}, & \left|u_{2}(\cdot)\right| \leq 1, \\
\dot{z}_{3}=z_{1}^{2}, & z(0)=0 \\
\dot{z}_{4}=z_{2}^{2}, & \varphi(z)=\left(z_{1}, z_{2}, z_{5}, z_{6}\right), \\
\dot{z}_{5}=z_{4} z_{1}^{2}-z_{1}^{7}+z_{1}^{10}+z_{2}^{10}, & \\
\dot{z}_{6}=z_{3} z_{2}^{2}-z_{2}^{7}+z_{1}^{10}+z_{2}^{10} . &
\end{array}\right.
$$

It is clear that the added 10th order terms do not at all affect the construction given in the proof of Theorem 3.2 and Corollary 3.4, and thus it is still true that the 8 th order cone is nonconvex. $b \geq 0$.

Proposition 5.1. For the system (5.1), $(0,0, a, b) \in \overline{\mathcal{K}}_{\varphi}^{8}$ if and only if $a \geq 0$ or

However, we will show that the perturbations destroy the controllability, and the tangent cones of all orders $m \geq 8$ are not convex.

TheOREM 5.2. For $0 \leq T<1$ the image $\varphi(\mathcal{R}(T))$ of the reachable set of system (5.1) does not contain any points of the form $\left(0,0, \eta_{3}, \eta_{4}\right)$ with $\eta_{3}<0$ and $\eta_{4}<0$.

Corollary 5.3. The system (5.1) is not STLOC about $y=0$.

Corollary 5.4. For the system (5.1) and $m \geq 8$, both $(0,0,-1,0) \in \overline{\mathcal{K}}_{\varphi}^{m}$ and $(0,0,0,-1) \in \overline{\mathcal{K}}_{\varphi}^{m}$ are generated by needle variations, but if $\eta_{3}, \eta_{4}<0$, then $\left(0,0, \eta_{3}, \eta_{4}\right) \notin \overline{\mathcal{K}}_{\varphi}^{m}$ for all $m>0$. In particular, for all $m>0$, the convex combination $\left(0,0,-\frac{1}{2},-\frac{1}{2}\right) \notin \overline{\mathcal{K}}_{\varphi}^{m}$.

Proof. We write $z$ and $x$ for the corresponding solutions of the perturbed and unperturbed systems (3.1) and (5.1); i.e., $z_{i}(t, u)=x_{i}(t, u)$ for $i=1,2,3,4$, while for $i=5,6$

$$
z_{i}(t, u)=x_{i}(t, u)+\int_{0}^{t}\left(x_{1}^{10}(\tau, u)+x_{2}^{10}(\tau, u)\right) d \tau \text { for all } 0 \leq t \leq T
$$

W.l.o.g. again assume that (3.15) holds (else permute in the following arguments the indices $(1,2,3,4,5,6) \mapsto(2,1,4,3,6,5)$ ). If $x_{5}(T, u) \geq 0$ (or $x_{6}(T, u) \geq 0$ ), then also $z_{5}(T, u) \geq 0$ (or $z_{6}(T, u) \geq 0$, respectively), and nothing needs to be shown. Thus suppose $x_{5}(T, u)<0$ and $x_{6}(T)<0$. Then use Lemma 3.7 and the key estimate (3.21) to obtain

$$
\int_{0}^{T} x_{2}^{10}(t) d t=\int_{0}^{T}\left|x_{2}(t)\right|^{10} d t \geq \frac{2}{11} \xi_{2}^{11} \geq \frac{2}{11}\left(\left(\frac{-x_{5}(T)}{T}\right)^{\frac{3}{35}}\right)^{11} \geq\left|x_{5}(T)\right|>0 .
$$

If $T<1$, clearly $\left|x_{5}(T)\right|<1$. Together with $\frac{33}{35}<1$ this shows that $z_{5}(T)=$ $x_{5}(T)+\int_{0}^{T}\left|x_{2}(t)\right|^{10} d t \geq 0$ if $z_{6}(T)<0$. 
For corresponding results for loss of controllability about a nonstationary reference trajectory, consider the system

$$
\left\{\begin{array}{lrl}
\dot{z}_{1}=u_{1}, & \left|u_{1}(\cdot)\right| \leq 1, \\
\dot{z}_{2}=u_{2}, & \left|u_{2}(\cdot)\right| \leq 1, \\
\dot{z}_{3}=z_{1}^{2}+\left(1+u_{01}\right), & \left|u_{01}(\cdot)\right| \leq 1, \\
\dot{z}_{4}=z_{2}^{2}+\left(1+u_{02}\right), & \left|u_{02}(\cdot)\right| \leq 1, \\
\dot{z}_{5}=z_{4} z_{1}^{2}-z_{1}^{7}+z_{1}^{10}+z_{2}^{10}, & z(0)=0, \\
\dot{z}_{6}=z_{3} z_{2}^{2}-z_{2}^{7}+z_{1}^{10}+z_{2}^{10}, & z^{*}(t)=(0,0, t, t, 0,0) .
\end{array}\right.
$$

By combining the arguments from the previous sections, one readily obtains the following statements. In particular, the 10th order terms in (5.4) do not affect the constructions of the 8th order tangent vectors; compare the proof of Theorem 3.2.

Proposition 5.5. For the system (5.4), $(0,0,0,0, a, b) \in \overline{\mathcal{K}}_{0}^{8}$ if and only if $a \geq 0$ or $b \geq 0$.

On the other hand the estimate (5.3) in the proof of Theorem 5.2 applies also to the system with nonstationary reference trajectory.

TheOREM 5.6. For $0 \leq T<1$ the reachable set $\mathcal{R}(T)$ of system (5.4) does not contain any points of the form $\left(0,0,0,0, q_{5}, q_{6}\right)$ with $q_{5}<0$ and $q_{6}<0$.

Corollary 5.7. The system (5.4) is not STLC about $z^{*}(t)=(0,0, t, t, 0,0)$.

The control variations constructed to generate the tangent vectors $(0,0,0,0,-1,0)$ and $(0,0,0,0,0,-1)$ satisfy the requirements of Definition 2.5. Together with Theorem 5.6 this allows us to state the last corollary for both notions of tangent vectors $\mathcal{K}_{0}^{m}$ and $\mathcal{K}_{T}^{m}$.

Corollary 5.8. For the system (5.4), $m \geq 8,0 \leq T<1$, none of the cones $\overline{\mathcal{K}}_{T}^{m}$ is convex. In particular, $(0,0,0,0,-1,0) \in \overline{\mathcal{K}}_{T}^{m}$ and $(0,0,0,0,0,-1) \in \overline{\mathcal{K}}_{T}^{m}$ are generated by needle variations at zero, but $\left(0,0,0,0,-\frac{1}{2},-\frac{1}{2}\right) \notin \overline{\mathcal{K}}_{T}^{m}$ for all $m>0$ and $T \geq 0$.

In summary, this section exploited the lack of convexity of tangent cones of order $8 \leq m<\frac{38}{3}$ to destroy the controllability properties of systems (3.1) and (4.1) by adding some perturbations, which at first view may appear to be of even higher order. While in the systems in the previous sections needle variations could still be combined to generate convex combinations of tangent vectors, albeit at a higher order, these perturbed systems make it entirely impossible to obtain convex combinations (at any order) of tangent vectors that are generated by needle variations.

6. Conclusion and further outlook. Summarizing, the analysis of the customdesigned systems in this article has yielded two major insights. The immediate result is a counterexample for a long-standing natural conjecture about needle variations.

- The most general notion of a tangent vector (which works well for stationary reference trajectories) does not necessarily yield convex cones either for nonstationary reference trajectories or for systems with output.

- Narrower notions of tangent vectors that yield convex cones, e.g., by requiring explicitly that needle variations must be movable by fixed times, may miss the controllability of some systems. (Recall in section 4 that the systems were controllable, but this cannot be detected with needle variations that require standard movability.)

- The common technical conditions on needle variations are not stated only because of not yet discovered stronger theorems, but they are essential even for some of the most benign systems. In a nutshell, the time(s) at which needle variations are generated must be movable - though not necessarily by a fixed distance, but by a distance of the same order of magnitude as the 
duration of the needle variation. Movability by a smaller amount, e.g., some power larger than one, is not sufficient; compare Remark 3.11. Otherwise, one should expect a lack of convexity of the cones of tangent vectors and a consequent failure to satisfy the hypotheses for open mapping theorems that are essential for making the cones into meaningful "approximating cones."

On a deeper level these examples cast further doubt on the structural stability and robustness of the STLC property. It is well known that simple Taylor approximations do not preserve controllability (see, e.g., [3]). For example, the system $\dot{x}_{1}=u$, $\dot{x}_{2}=x_{1}, \dot{x}_{3}=x_{2}^{2}+x_{1}^{3}[21]$ is STLC about $x(0)=0$ and $|u(\cdot)| \leq 1$, but its quadratic Taylor approximation $\dot{y}_{1}=u, \dot{y}_{2}=y_{1}, \dot{y}_{3}=y_{2}^{2}$ is not STLC. Instead, many controllability results of the past two decades rely on nilpotent approximating systems [4, 12] which are based on graded structures: For a fixed set of local coordinates $\left(x_{1}, \ldots, x_{n}\right)$ on $\mathbb{R}^{n}$ and weights $r_{1}, \ldots, r_{n} \geq 1$, consider the family of dilations $\Delta: \mathbb{R}^{+} \times \mathbb{R}^{n} \mapsto \mathbb{R}$ defined by $\Delta_{s}(x)=\left(s^{r_{1}} x_{1}, \ldots, s^{r_{n}} x_{n}\right)$. A function $\varphi: \mathbb{R}^{n} \mapsto \mathbb{R}^{n}$ is called homogeneous of order $m$ with respect to $\Delta$ if $\varphi \circ \Delta_{s}=s^{m} \varphi$. A vector field $f$ is called homogeneous of order $k$ if (considered as a first order partial differential operator) it maps (smooth) homogeneous functions of any order $m$ to homogeneous functions of order $m+k$. To approximate the system (2.1) by a nilpotent system of the same form, replace each vector field $f_{i}$ by the principal part $g_{i}$ of the expansion of $f_{i}$ into a (generally infinite) series of homogeneous vector fields. If all vector fields $g_{i}$ have negative degrees of homogeneity, then all are polynomial vector fields of cascade form, and they generate a nilpotent Lie algebra; see the survey [12] for details. Roughly speaking, the fundamental result is that if such homogeneous nilpotent approximating system with vector fields $g_{i}$ is STLC, then the original system is $S T L C$; see, e.g., [4, 22]. By varying the construction of the local coordinates and dilation exponents $r_{i}$, several major results for STLC follow; see, e.g., [4, 21, 22]. On the other hand, it is known that there exist polynomial systems that are STLC, but for them any such homogeneous nilpotent approximating system is not STLC [15].

Returning to the systems (3.1) and (4.1) discussed in this article which are STLOC and STLC, respectively, these are similar to the system in [15], as any of the standard nilpotent approximation schemes will simply delete the terms $x_{1}^{7}$ and $x_{2}^{7}$ on the right-hand side, yielding homogeneous nilpotent approximating systems that are not STLOC nor STLC. Since the example constructed in [15], one has been searching for more general approximating schemes that would recognize the need to preserve terms such as $x_{1}^{7}$ and $x_{2}^{7}$ in the above systems, which are essential for their controllability and which would only truncate truly higher order terms that are not essential for controllability. However, the analysis in the preceding section of the systems (5.1) and (5.4) raises new questions as the inclusion of the even higher order terms $x_{1}^{10}$ and $x_{2}^{10}$ again causes loss of controllability. One naturally wonders whether it is possible to construct such an infinite chain, which could possibly lead to a negative answer to the long-standing question of whether STLC (of analytic systems) is finitely determined [2].

Acknowledgments. The authors thank the anonymous referees and H. Frankowska who made numerous suggestions to help improve the article.

\section{REFERENCES}

[1] A. Agrachev, Newton diagrams and tangent cones to attainable sets, in Analysis of Controlled Dynamical Systems, Progr. Systems Control Theory 8, B. Bonnard, B. Bride, J. P. Gauthier, and I. Kupka, eds., Birkhäuser Boston, Boston, MA, 1991, pp. 11-20. 
[2] A. Agrachev, Is it possible to recognize local controllability in a finite number of differentiations?, in Open Problems in Mathematical Systems and Control Theory, V. Blondel, E. Sontag, M. Vidyasagar, and J. Willems, eds., Springer, London, 1999, pp. 15-18.

[3] R. M. Bianchini and G. Stefani, Normal local controllability of order one, Internat. J. Control, 39 (1984), pp. 701-714.

[4] R. M. BIANCHINI AND G. STEFANi, Graded approximations and controllability along a trajectory, SIAM J. Control Optim., 28 (1990), pp. 903-924.

[5] R. M. Bianchini and G. Stefani, Controllability along a trajectory: A variational approach, SIAM J. Control Optim., 31 (1993), pp. 900-927.

[6] R. M. Bianchini, Good needle-like variations, in Proc. Sympos. Pure Math. 64, AMS, Providence, RI, 1999, pp. 91-101.

[7] R.-M. BIANCHINI AND M. KAWSKI, Lack of convexity for tangent cones of needle variations, in Proceedings of the 41st IEEE Conference on Decision and Control, Vol. 2, IEEE Press, Piscataway, NJ, 2002, pp. 1916-1921.

[8] A. Bressan, A high-order test for optimality of bang-bang controls, SIAM J. Control Optim., 23 (1985), pp. 38-48.

[9] H. Frankowska, An open mapping principle for set-valued maps, J. Math. Anal. Appl., 127 (1987), pp. 172-180.

[10] H. FranKowsKa, Local controllability of control systems with feedback, J. Optim. Theory Appl., 60 (1989), pp. 277-296.

[11] H. Frankowska, A conical open mapping principle for set-valued maps, Bull. Austral. Math. Soc., 45 (1992), pp. 53-60.

[12] H. Hermes, Nilpotent and high-order approximations of vector field systems, SIAM Rev., 33 (1991), pp. 238-264.

[13] B. KAskosz, Abundant subsets of generalized control systems, in Systems Modelling and Optimization, Chapman \& Hall/CRC. Res. Notes. Math. 396, Boca Raton, FL, 1999, pp. 108116.

[14] M. KAWskI, An angular open mapping theorem, in Analysis and Optimization of Systems, Lect. Notes in Control and Inform. Sci. 111, A. Bensoussan and J. L. Lions, eds., Springer, Berlin, 1988, pp. 361-371.

[15] M. Kawski, Control variations with an increasing number of switchings, Bull. Amer. Math. Soc., 18 (1988), pp. 149-152.

[16] H. Knobloch, Higher Order Necessary Conditions in Optimal Control Theory, Lect. Notes in Control and Inform. Sci. 34, Springer-Verlag, Berlin-New York, 1981.

[17] A. J. KRENER, The high order maximal principle and its application to singular extremals, SIAM J. Control Optim., 15 (1977), pp. 256-293.

[18] E. Lee And L. Markus, Foundations of Optimal Control Theory, John Wiley, New York, 1967.

[19] L. Pontryagin, V. Boltyanskit, R. Gamkrelidze, and E. Mischenko, The Mathematical Theory of Optimal Processes, John Wiley, New York, 1962.

[20] G. Stefani, Polynomial approximations to control systems and local controllability, in Proceedings of the 25th IEEE Conference on Decision and Control, IEEE Press, Piscataway, NJ, 1985, pp. 33-38.

[21] H. J. Sussmann, Lie brackets and local controllability: A sufficient condition for scalar-input systems, SIAM J. Control Optim., 21 (1983), pp. 686-713.

[22] H. J. Sussmann, A general theorem on local controllability, SIAM J. Control Optim., 25 (1987), pp. $158-194$.

[23] H. Sussmann, Needle variations and almost lower semicontinuous differential inclusions, SetValued Anal., to appear. 